\title{
Modelling the behaviour of sensitive clays experiencing large deformations using non-local regularisation techniques
}

\author{
Vikram Singh $^{\mathrm{a}, *}$, Sam Stanier $^{\mathrm{b}}$, Britta Bienen ${ }^{\mathrm{a}}$, Mark F. Randolph ${ }^{\mathrm{a}}$ \\ ${ }^{a}$ Centre for Offshore Foundation Systems, University of Western Australia, Perth, WA \\ 6009, Australia. \\ ${ }^{b}$ Cambridge University Engineering Department, Trumpington Street, Cambridge, CB2 \\ $1 P Z, U K$.
}

\begin{abstract}
This paper presents a methodology to simulate the strain-softening-hardening response of sensitive clays when subjected to extensive remoulding. A nonlocal strain-softening technique is implemented into the Abaqus Finite Element Analysis (FEA) software through its user defined material subroutine UMAT, in the form of a critical state based strain-softening constitutive model. For the comparison of conventional and non-local formulations, a series of analyses have been performed investigating mesh dependency issues and the validity of a simple softening-scaling rule for practical applications. The non-local model significantly reduces solution mesh-dependency for small-strain analyses of biaxial shear and updated Lagrangian analyses of buried pipe uplift. Finally, the non-local model is applied to simulate undrained cycles of penetration and the consolidated-undrained capacity of a T-bar in kaolin clay. This analysis highlights the limitations of the use of standard element tests (e.g. triaxial tests) to calibrate the model input parameters that control the strain-softening aspect of the constitutive law, and the need for a constitutive law that captures the partial recovery of sensitivity during consolidation.
\end{abstract}

Keywords: Sensitive clays, Strain localisation, Non-local regularisation, Large deformations, Buried pipe uplift, T-bar penetration tests

\footnotetext{
${ }^{*}$ Corresponding author

Email address: vikram.singh@research.uwa.edu.au (Vikram Singh)
} 


\section{Introduction}

Fine-grained offshore sediments exhibit strain-softening behaviour with the development of excess pore pressures during the large near-undrained deformations commonly encountered in geotechnical processes such as full-flow penetrometer testing, anchor installation, keying and pullout, pipe laying and buckling. The subsequent dissipation of excess pore pressures induces strain-hardening of the soil and results in partially recovered or even increased strength. It is well established in the literature that numerical modelling of the strain-softening response using classical continuum models such as the finite element method can be challenging due to severe mesh dependent strain localisation $[1,2]$, which usually appears in the form of shear bands with the thickness of a single element. Due to absence of an internal length scale, these models fail to describe the size effect of strain localisation correctly resulting in mesh dependent solutions [2].

Several regularisation or mitigation techniques have been reported in the literature that can ensure that finite element simulation output remains independent of the mesh discretisation when rate-independent elastoplastic strain-softening models are adopted to describe the material response. The most common mitigation methods involve the implementation of Cosserat continuum methods [3, 4], gradient dependent continuum methods $[5,6,2]$ and integral-type non-local strain methods $[7,8,9]$. These techniques all introduce an internal length scale to the constitutive model formulation, which controls the degree of deformation localisation and preserves the well posedness of the governing partial differential equations irrespective of the refinement of the mesh [2].

The simple Tresca-derived elastic-perfectly plastic constitutive model with strain-softening, proposed by Einav and Randolph [10], has been used extensively to simulate ultra large deformation problems such as T-bar penetration in soft clays $[11,12,13,14]$. In these works, mesh dependency has typically been mitigated by the incorporation of viscous (or rate) effects in the constitutive equations, which enhances the undrained shear strength of the soil by approximately $5-20 \%$ per log cycle of rate over the typical laboratory strain rate of $1 \%$ /hour $[15,16,10]$. However, the efficacy of this approach in mitigating mesh dependency reduces with reducing strain rate. In other words the transition from undrained to drained behaviour is not 
likely to be properly regularised by such an approach. This transition occurs many times in what might be classed as episodic undrained problems, such as T-bar penetration or pipeline buckling, which involve undrained displacement followed by cycles of consolidation and further undrained displacements $[17,18]$. These problems require a constitutive model with the capability to model both strain-softening and strain-hardening, as well as a regularisation approach that is effective, irrespective of the operative strain rate.

The main objective of this paper is to demonstrate the application of a regularised large deformation FE based technique for use in the modelling of strain-softening-hardening behaviour. For simplicity, and in order to validate the efficacy of the non-local regularisation approach implemented directly, viscous effects were not considered in this study. The integral-type nonlocal strain-softening approach [19] was adopted to regularise FE solutions using a simple strain-softening extension of the classical Modified Cam Clay constitutive model [20]. The model was implemented in the commercial finite element software Abaqus, via its user-defined material subroutine UMAT.

The mesh-dependency of the finite element (FE) solutions obtained using the local and non-local models is first demonstrated through a series of small strain biaxial compression simulations. The implementation is then used to simulate a boundary value problem in the form of buried pipe uplift via Large Deformation Finite Element (LDFE) analyses using the Remeshing and Interpolation Technique with Small Strains (RITSS; Hu and Randolph [21]) approach, in order to further explore the mesh dependency when the mesh is periodically updated in an updated-Lagrangian sense [12]. The results obtained using the non-local model are shown to be insensitive to the mesh topology. Perhaps surprisingly, the computational efficiency of the analyses appears largely unaffected, with the additional computational burden of the non-local calculations being offset by an improvement in the rate of convergence due to the preservation of convexity of the governing partial differential equations [2]. Finally, undrained and consolidated-undrained cycles of T-bar penetration in kaolin clay are modelled using the same approach, with the results compared to T-bar penetration experiments. This final analysis illustrates: (i) the difficulty in selecting appropriate constitutive parameters to control the rate of strain-softening using element test data; and (ii) the need for constitutive models that capture the recovery of sensitivity after consolidation induced hardening. 


\section{A simple constitutive model for strain-softening-hardening}

The Structured Modified Cam Clay (SMCC) constitutive model proposed by Mašín [20] has been adopted in this paper. This is a simple elastoplastic critical state model for structured fine-grained soils based on the classical Modified Cam Clay (MCC) [22]. This model was chosen because it is the simplest variant of the Cam Clay genera of constitutive model that can capture both the strain-softening and strain-hardening aspects of soil behaviour and is straightforward to implement in updated-Lagrangian methods such as the RITSS approach.

\subsection{Local formulation}

The model requires a total of nine parameters, which can be grouped into two distinct groups: (i) the basic parameters $M, \lambda^{*}, \kappa^{*}, N$ and $G$ (or $\nu$ ); and (ii) the strain-softening parameters $k, A, S_{t}$ and $S_{f}$. The basic parameters have physical interpretations that are consistent with the original MCC model parameters, except that the so-called Butterfield compression law [23], replacing the conventional $e-\ln p^{\prime}$ relationship of MCC model by $\ln (1+e)-\ln p^{\prime}$, has been adopted. The second group of parameters control the strain softening aspect of the model response. The model has two state variables: void ratio $e$ and sensitivity $s_{e p}$. The shape of the yield surface $(f)$ is expressed as follows:

$$
f=q^{2}+M^{2} p^{\prime}\left(p^{\prime}-s_{e p} p_{c}\right)
$$

where $p^{\prime}$ and $q$ are mean effective stress and deviatoric stress respectively, $p_{c}$ represents the yield surface size of the reference soil, where it can be seen that the sensitivity state variable $s_{e p}$ acts as a simple scalar multiplier on the size of the yield surface. The initial magnitude of the sensitivity state variable $s_{\text {epi }}$ can be derived from the slopes of the normal consolidation line (NCL) $\lambda^{*}$, unload-reload line (URL) $\kappa^{*}$ and the initial sensitivity $S_{t}$ as follows:

$$
s_{\text {epi }}=S_{t}^{\left(\frac{\lambda^{*}}{\lambda^{*}-\kappa^{*}}\right)}
$$

Similarly, the value of the sensitivity state variable for the reference soil (typically taken as unity) $s_{e p f}$ is calculated by:

$$
s_{e p f}=S_{f}^{\left(\frac{\lambda^{*}}{\lambda^{*}-\kappa^{*}}\right)}
$$


The relation in equation 2 represents the ratio of the yield envelope size for the intact and the reference (i.e. remoulded) soil states. Associated flow is assumed for simplicity, hence the plastic potential surface is identical to the yield surface given in Equation 1. The void ratio $e$ is related to the quantity $p_{c}$ through:

$$
\ln (1+e)=N-\kappa^{*} \ln p^{\prime}-\left(\lambda^{*}-\kappa^{*}\right) \ln \left(\frac{p_{c}}{p_{r}}\right)
$$

where $p_{r}$ is the reference pressure $1 \mathrm{kPa}$.

The incremental strain rate drives the evolution of the state variables $e$ and $s_{e p}$ according to:

$$
\begin{gathered}
\dot{e}=-(1+e) \dot{\varepsilon}_{\mathrm{v}} \\
\dot{s}_{e p}=-\frac{k}{\left(\lambda^{*}-\kappa^{*}\right)}\left(s_{e p}-s_{e p f}\right) \dot{\varepsilon}_{s}
\end{gathered}
$$

where $k$ controls sensitivity degradation rate, $\dot{\varepsilon}_{v}$ is the volumetric strain rate and $\dot{\varepsilon}_{s}$ is the equivalent plastic strain rate obtained from the plastic volumetric and the deviatoric strain rates (i.e., $\dot{\varepsilon}_{v}^{p}$ and $\dot{\varepsilon}_{q}^{p}$, respectively) by:

$$
\dot{\varepsilon}_{s}=\sqrt{\left(\dot{\varepsilon}_{v}^{\mathrm{p}}\right)^{2}+\frac{A}{1-A}\left(\dot{\varepsilon}_{q}^{\mathrm{p}}\right)^{2}}
$$

The parameter $A$ governs the relative contribution of $\dot{\varepsilon}_{v}^{p}$ and $\dot{\varepsilon}_{q}^{p}$ driving the strength degradation. In the present work, a slightly different form of the equation 7 (previously used in several strain-softening models including Taiebat et al. [24]) has been adopted to facilitate greater flexibility as it allows the value of $A$ to be set to unity in order to prescribe softening driven purely by plastic shear strain, writing the plastic strain rate as:

$$
\dot{\varepsilon}_{s}=\sqrt{(1-A)\left(\dot{\varepsilon}_{v}^{\mathrm{p}}\right)^{2}+A\left(\dot{\varepsilon}_{q}^{\mathrm{p}}\right)^{2}}
$$

In this model formulation, the local incremental plastic strains drive the strain-softening aspect of the model response, which is why it is referred to herein as a 'local' formulation. 


\subsection{Regularisation method: non-local softening}

The regularised variant of the model simply uses a non-locally weighted measure of the volumetric and deviatoric strains, $\dot{\varepsilon}_{v}^{p}$ and $\dot{\varepsilon}_{q}^{p}$, to drive the softening response, similar to Galavi and Schweiger [25], Summersgill et al. [26], Mánica et al. [27], and Monforte et al. [28]. In this method, the strainsoftening at a material point is linked to the state of plastic deformation in the material points surrounding the current integration point, rather than being solely a function of the strain at the current integration point. The basic constitutive relations remain unchanged and it is therefore relatively straightforward to implement the required modifications into LDFE simulations compared to other regularisation methods. Technically, since only the variable controlling strain-softening response is considered non-local, this technique is referred to as a partially non-local approach; fully non-local variants of this approach utilise non-local stresses as well as strains in the constitutive response [25].

In the local SMCC model, the equivalent plastic strain expressed by Equation 8 governs the strain-softening response at a FE integration point (IP). In the non-local SMCC model, this value is replaced by a spatially averaged value of the same quantity, $\bar{\varepsilon}_{s}$, calculated as:

$$
\bar{\varepsilon}_{s}\left(\mathrm{X}_{i}\right)=\int_{V} \alpha\left(\mathrm{X}_{i}, \mathrm{X}_{j}\right) \dot{\varepsilon}_{s}\left(\mathrm{X}_{j}\right) d V
$$

where $X_{i}$ and $X_{j}$ are positions of any IP under consideration and its neighbouring IPs, respectively, $d V$ is the volume of the element represented by each integration point and $\alpha\left(\mathrm{X}_{i}, \mathrm{X}_{j}\right)$ is a normalised weight function used in spatial averaging of local variable $\dot{\varepsilon}_{s}$ at all integration points around the IP at which the stress state is being updated. The normalised form of the function preserves the uniform non-local distribution of strains when the local distribution is uniform and is expressed by:

$$
\alpha\left(\mathrm{X}_{i}, \mathrm{X}_{j}\right)=\frac{\alpha_{0}\left(\left\|\mathrm{X}_{i}-\mathrm{X}_{j}\right\|\right)}{\int_{V} \alpha_{0}\left(\left\|\mathrm{X}_{i}-\mathrm{X}_{j}\right\|\right) d V}
$$

where $\alpha_{0}\left(\left\|\mathrm{X}_{i}-\mathrm{X}_{j}\right\|\right)$ is the weight function value that controls relative contribution of any IP in strain softening. The value of $\alpha_{0}$ depends on assumed shape of the weight function and distance between two IPs (i.e. $\left.\left\|\mathrm{X}_{i}-\mathrm{X}_{j}\right\|\right)$. Various weighting schemes have been proposed to determine the non-local strains from the local strain, including Gaussian [29], over 
non-local [29] and bi-modal functions [25]. In this study, both Gaussian and bi-model functions were implemented, given respectively by:

$$
\begin{gathered}
\alpha_{0}=\frac{1}{l_{c} \sqrt{\pi}} e^{-\left(\frac{\left\|x_{i}-x_{j}\right\|}{l_{c}}\right)^{2}} \\
\alpha_{0}=\frac{\left\|X_{i}-X_{j}\right\|}{l_{c}^{2}} e^{-\left(\frac{\left\|x_{i}-x_{j}\right\|}{l_{c}}\right)^{2}}
\end{gathered}
$$

in which the parameter $l_{c}$ - typically referred to as the 'characteristic length' - introduces a length scale dependency into the constitutive response in order to indirectly control the size of the strain localisation features (or shear bands). Figure 1 plots both weight functions against distance between two IPs for an arbitrary characteristic length of one metre. It can be seen that the Gaussian function assumes a maximal contribution from the IP under consideration, whereas the bi-modal function suggested by Galavi and Schweiger [25] (hereafter referred to as the 'G\&S method' following Summersgill et al. [26]) ignores the local equivalent strain at the current integration point. The non-local averaging calculations can only be guaranteed to be effective if the characteristic length, $l_{c}$, takes a value greater than the largest element size in the FE mesh discretisation.

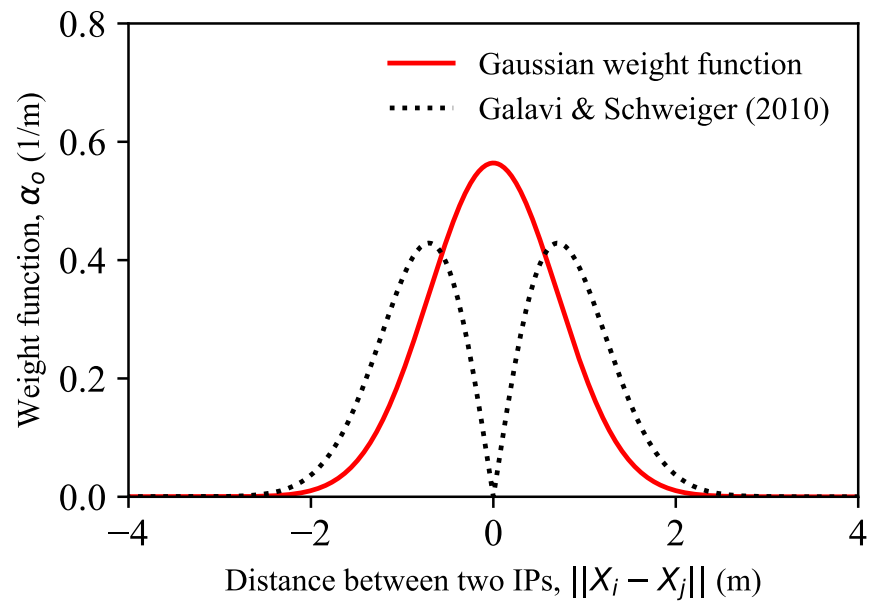

Figure 1: Comparison of non-local weighting functions for $l_{c}=1.0 \mathrm{~m}$. 


\subsection{Finite element implementation}

The local SMCC model has been implemented in the commercial FE code Abaqus [30] via its user specified-material subroutine or UMAT. It was written in Fortran, based on the implementation of Gudehus et al. [31], which uses an adaptive Runge-Kutta-Fehlberg method (RKF23) for the stress integration, with yield surface drift correction following the procedure described by Sloan et al. [32]. The non-local aspect of the model has been implemented by extending the UMAT subroutine for the local model, following the methodology suggested by Chow et al. [33]. To implement the weighted spatial averaging in the numerical algorithm, equivalent discrete integrals of equations 9 and 10 are adopted which can be expressed as:

$$
\begin{gathered}
\bar{\varepsilon}_{s}\left(X_{i}\right)=\sum_{j=1}^{N I P} \alpha_{i j} \dot{\varepsilon}_{s}\left(X_{j}\right) \\
\alpha_{i j}=\frac{\alpha_{0}\left(\left\|X_{i}-X_{j}\right\|\right)}{\sum_{k=1}^{N I P} \alpha_{0}\left(\left\|X_{i}-X_{k}\right\|\right)}
\end{gathered}
$$

where NIP is the total number of integration points in the adopted domain discretisation. When performing non-local spatial averaging of the plastic strains at any IP, access to information of the current state of all IPs in the simulation is required. To perform constitutive calculations, Abaqus successively calls the UMAT subroutine at all of the integration points of the elements for which the material definition includes a user-specified constitutive response. The UMAT interface allows access to state information (e.g., stresses, strain increment, step time, position coordinates and other state-dependent variables) of the IP currently under consideration but not all IPs in the model. This is the most critical issue with regards to the implementation of the non-local calculations in a UMAT subroutine. Previously, the access to global state information has been gained by combining two or more subroutines, specifically UMAT (for constitutive response calculations) and UEL (a user-element subroutine to implement a user-defined element) [34].

Chow et al. [33] suggested a simpler approach which does not require the use of the UEL subroutine to access the state details of neighbourhood IPs. In this method, a three dimensional FORTRAN storage array called a COMMON BLOCK was employed to store the information required for the non-local implementation (coordinates of IPs and the local 
strain variables). These details were stored at the beginning of the analysis step and then updated at the end of every increment. In this paper, a method similar to this approach has been used. However, instead of using a COMMON BLOCK array, which limits the storage size to maximum of 2GB, Abaqus utility routines are used to create global allocatable arrays. At the time of the array creation in a subroutine, an identifier number (i.e a user defined arbitrary integer) is assigned to each array which can then can be used to access the array data in any other subroutine. Four such arrays (EIXYS[3,NIPP,NUEL], IPID[NEGBR,NIPP,NUEL], ELMID[NEGBR,NIPP,NUEL], and WEGHT[NEGBR,NIPP,NUEL]) were created to store and transfer the required information for the non-local strainsoftening calculations. In these arrays, NIPP is the number of total IPs per element, NUEL is the total number of elements in the mesh and NEGBR is the maximum number of $\mathrm{K}$ nearest neighbouring IPs for any IP in the model. In the first Abaqus call to the UMAT subroutine, the coordinates of all of the IPs are stored in the first and second dimensions of EIXYS. The third dimension is used to store the local strain-softening variable which is updated at the end of every increment. The non-local averaging is a computationally expensive process and therefore, for computational efficiency, $\mathrm{K}$ nearest neighbours (KNN) of all IPs are identified in the first step increment based on the the relative distance between them, and their identifier numbers (IP and element number in FE mesh) are stored in the arrays IPID and ELMID. Then, the averaging weights are calculated based on the characteristic length and are stored into the array WEGHT[NEGBR,NIPP,NUEL] for all integration points. In this way, the identification of KNNs and calculation of their respective weights are performed once for each small strain step of the analysis and simply accessed from the storage array to perform non-local averaging during each increment.

\subsection{Material parameters and model validation}

SMCC model parameters for Pisa clay and UWA kaolin clay were adopted from Mašín [20] and Ragni et al. [35], respectively. These were previously calibrated using standard geotechnical element tests performed on natural Pisa and reconstituted kaolin clay samples and the various parameters adopted are presented in Table 1. For the Pisa clay, the identification of the parameters $\phi_{c}$ or $M, N^{*}, \lambda^{*}$ and $\kappa^{*}$ was done by simulating isotropic compression tests on reconstituted clay, and the softening parameters $S_{t}, k$ and $A$ were found by the direct evaluation of the results of experiments on natural Pisa clay, 
using the structure degradation law of the model[20]. The Poisson's ratio of the soil was assumed to be 0.3 , rather than prescribing a constant shear modulus as required in the original model equations. In Ragni et al. [35], the basic parameters of the hypoplastic clay model (of which the SMCC model is an elastoplastic equivalent) for the UWA kaolin clay were adopted as the MCC model parameters defined by Stewart [36], with appropriate conversion from $e-\ln p^{\prime}$ to $\ln (1+e)-\ln p^{\prime}$ space. The initial sensitivity $S_{t}$ was adopted as T-bar based sensitivity $S_{T-b a r}$, obtained from the cyclic T-bar tests in kaolin clay. The softening parameter $k$ was back calculated from the triaxial compression tests presented in Figure 2, and the value of the parameter $A$ was assumed as 0.2 .

The triaxial compression tests on UWA kaolin clay [35] were simulated using the SMCC model to demonstrate the model capabilities and validate the model implementation in Abaqus. Figure 2 shows that the slope of the critical state line $M$ was approximately $0.80\left(\phi_{c}=20.6^{\circ}\right)$ for the tests with mean effective stresses at the end of isotropic consolidation of $100 \mathrm{kPa}$ and $400 \mathrm{kPa}$, and $0.90\left(\phi_{c}=23.0^{\circ}\right)$ for the test with mean effective stress of $50 \mathrm{kPa}$. The stress paths deviate from the CSL at approximately $10 \%$ axial strain, which is likely due to the onset of strain localisation so the data beyond this point has been faded out. The upper bound of $M=$ 0.9 derived from the test with lowest initial $p^{\prime}$ is close to the value of 0.92 $\left(\phi_{c}=23.5^{\circ}\right)$ reported by Stewart [36], which has been adopted extensively in past numerical studies([37],[38],[39],[40],[41]). Considering the effective stress levels important for the boundary value problem simulation presented later in this study (i.e. $\approx 50 \mathrm{kPa}$ ), the critical state friction angle of $23.0^{\circ}$, also used by Ragni et al. [35], was adopted in this study. 
Table 1: Parameters for Pisa and UWA Kaolin clay, after Mašín [20] and Ragni et al. [35], respectively

\begin{tabular}{|c|c|c|}
\hline Parameter & Pisa clay & Kaolin clay \\
\hline \hline Slope of NCL, $\lambda^{*}$ & 0.14 & 0.0811 \\
Slope of URL, $\kappa^{*}$ & 0.02 & 0.019 \\
Poisson's ratio, $\nu$ & 0.3 & 0.3 \\
Frictional constant, $M$ & 0.85 & 0.90 \\
Intercept of the NCL at $p^{\prime}=1 \mathrm{kPa}, N^{*}$ & 1.56 & 1.195 \\
\hline Initial sensitivity, $S_{t}$ & 3.45 & 2.2 \\
Final sensitivity, $S_{f}$ & 1.0 & 1.0 \\
Degradation rate, $k$ & 0.4 & 0.2 \\
Degradation ratio, $A$ & 0.1 & 0.2 \\
\hline
\end{tabular}

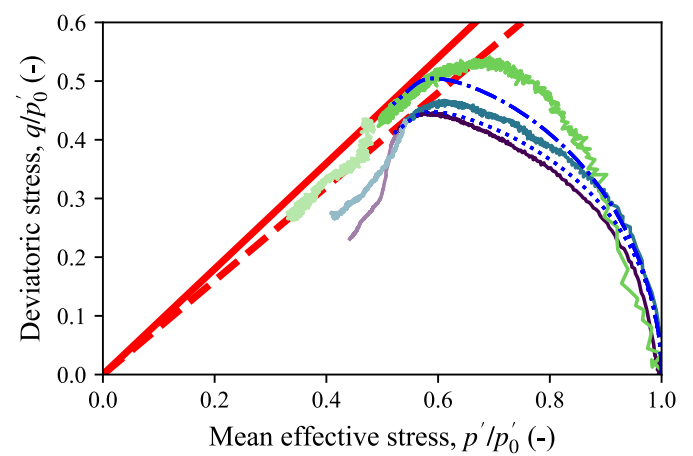

(a)

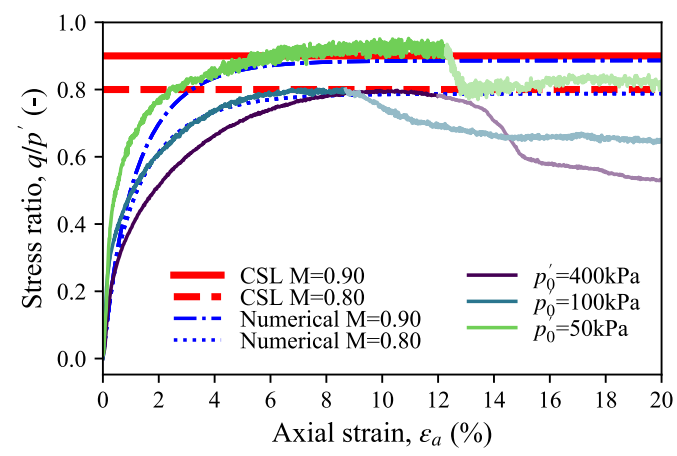

(b)

Figure 2: Comparison of experimental and numerical data of Triaxial undrained compression tests on kaolin clay: (a) effective stress path in $q / p_{0}^{\prime}-p^{\prime} / p_{0}^{\prime}$ space, and (b) stress ratio variation with axial strain in $q / p^{\prime}-\varepsilon_{a}$ space.

\section{Simulation of a classical element test: biaxial compression}

\subsection{Analyses details}

Classical biaxial compression simulations have previously been used to demonstrate the mesh dependency of local strain-softening models and efficacy of the non-local techniques in regularising FE solutions involving strainsoftening [25, 42, 27, 28]. In this work, a series of undrained and drained small strain FE analyses have been performed for biaxial compression using 
the local and the non-local strain-softening methods. All the analyses were performed using plane strain models generated in Abaqus. The geometry, typical undeformed mesh and boundary conditions of the test are illustrated in Figure 3. The soil specimen was considered to be a square domain of $2 \mathrm{~m} \times 2 \mathrm{~m}$ size. Only $1 / 4$ of the area of the domain was modelled due to the symmetrical boundary conditions. The left hand side boundary of the model (i.e. the vertical axis of symmetry) was allowed to move in the vertical direction but was restrained in the horizontal direction. Along the bottom boundary (i.e. the horizontal axis of symmetry) horizontal displacement was allowed with vertical movement restrained. The top boundary of the mesh was restrained against any horizontal movement and vertical displacement was prescribed to apply the compression to the specimen, which is sufficient to create strain localisation [42].

For domain discretisation, four meshes with 100 (10 x 10), 400 (20 x 20), 900 (30 x 30) and 1600 (40 x 40) eight node quadratic plain strain quadrilateral elements (CPE8RP in the Abaqus Standard library) were used. Figure 3 shows the mesh created for the case with 100 (10 x 10) elements numbered from 1 to 10 along horizontal and vertical sides. To establish the initial stress state in the model, vertical and horizontal stresses of $50 \mathrm{kPa}$ and $100 \mathrm{kPa}$ were applied at the top and the right side boundaries of the mesh respectively. The specimen compression response was obtained using displacement controlled analyses which were performed by applying a $0.1 \mathrm{~m}$ vertical displacement at the top boundary of the mesh. The input SMCC model parameters for the Pisa clay were used. The total step time was prescribed such that no dissipation of generated excess pore pressure occurs in the undrained analyses, while full drained conditions were maintained during drained analyses. The characteristic length was assumed to be $0.1 \mathrm{~m}$ (i.e. equal to the element size in the coarsest mesh) in all of the non-local analyses. 


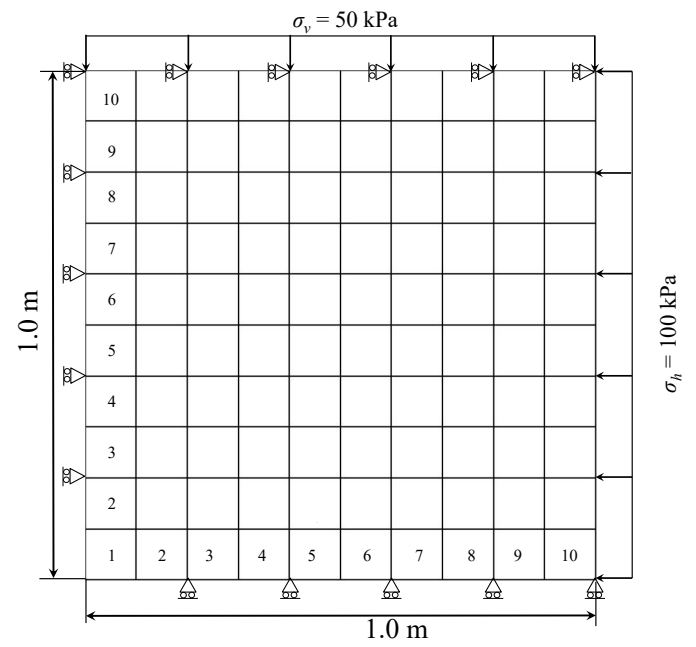

Figure 3: Illustration of analysis mesh used for biaxial simulations.

\subsection{Results: mesh dependency}

The vertical reaction force at the top of the analysis mesh is plotted against applied vertical displacement $\left(\delta_{v}\right)$ in Figure 4 for all of the considered cases. Figure 5 plots contours of the degree of soil remoulding (i.e. the ratio of current and initial values of the sensitivity state variable $s_{e p} / s_{e p i}$ ) at the maximum compression of the mesh. The responses obtained from the local undrained simulations is shown in Figure 4a. The force-displacement curves are identical until the peak resistance is reached, after which an increasing rate of strain-softening is clearly observed with an increase in the number of elements. This is due to mesh dependent strain localisation, where the shear band thickness is proportional to the size of a single element (compare sensitivity distribution contours in Figures 5a, d, g, and j). Unlike the local undrained analyses, the local drained analyses do not suffer from the mesh dependency problem (see Figure 4b). In drained conditions, the dissipation of generated excess pore pressure causes strain-hardening of the soil, which is sufficient to override the strain-softening effects. The results for the undrained analyses using the non-local methods (Gaussian and G\&S methods) are presented in Figures $4 \mathrm{c}$ and d. The strain-softening rate remains almost identical for all mesh densities. This demonstrates that the non-local strain-softening methods alleviate the mesh dependency. The size of the strain-softened zone and degree of remoulding is similar for the different discretisations considered in this work (compare Figures $5 \mathrm{~b}, \mathrm{e}, \mathrm{h}$ and 
$\mathrm{k}$ and Figures 5c, f, i and l). For the non-local Gaussian method, a small mesh dependency persists and the size of the strain-softening zone appears to reduce with decreasing element size. The non-local G\&S method appears to regularise the simulations more effectively, which is consistent with the conclusion reached by previous research [43], and so was adopted for all the boundary value problem analyses performed in this study hereafter.

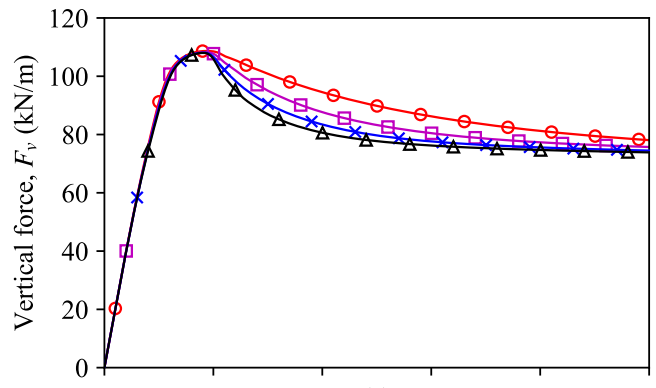

(a)

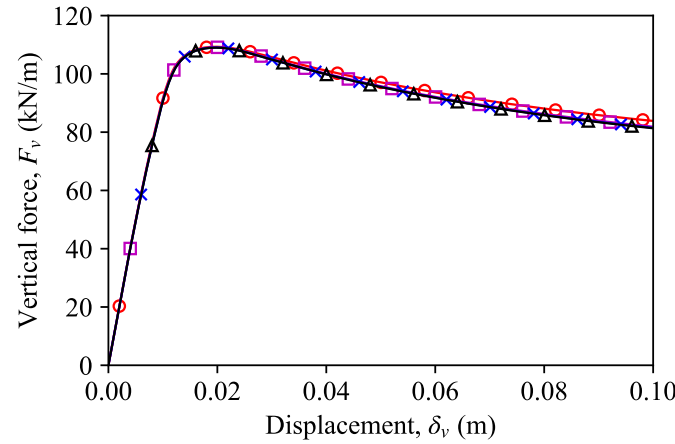

(c)

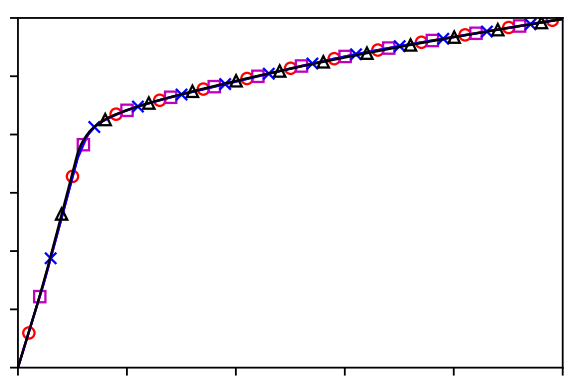

(b)

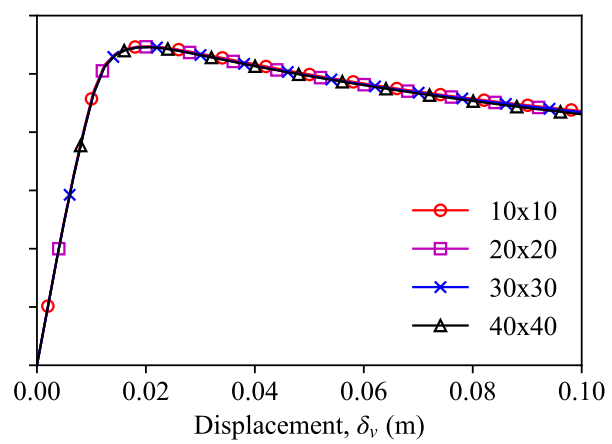

(d)

Figure 4: Small-strain simulation response for varying mesh density: (a) local undrained; (b) local drained; (c) non-local (Gaussian) undrained; and (d) non-local (G\&S) undrained. 


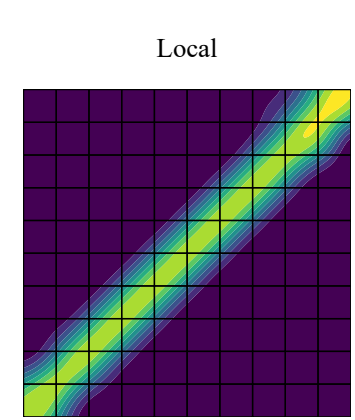

(a)

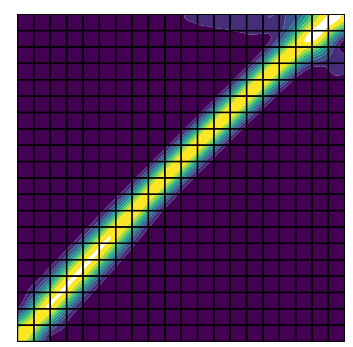

(d)

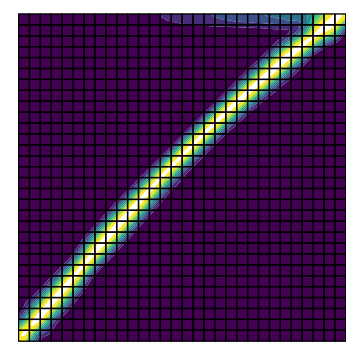

(g)

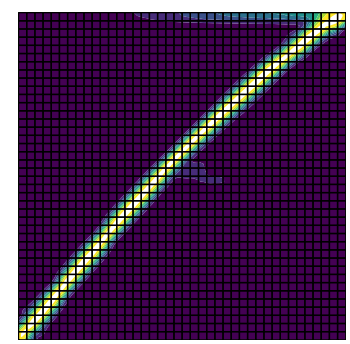

(j)

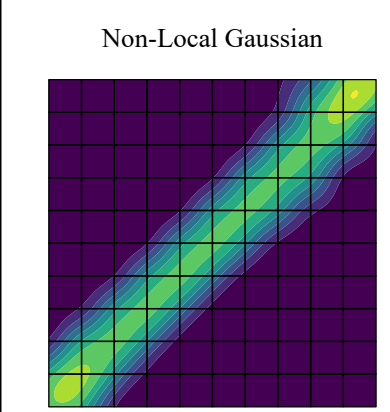

(b)

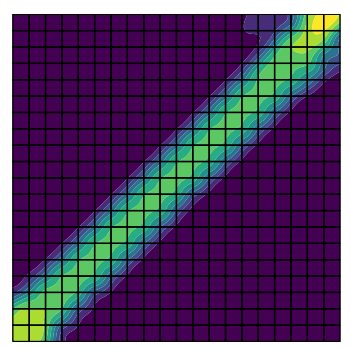

(e)

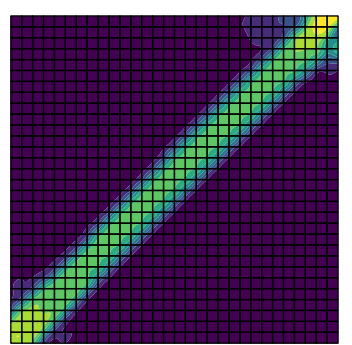

(h)

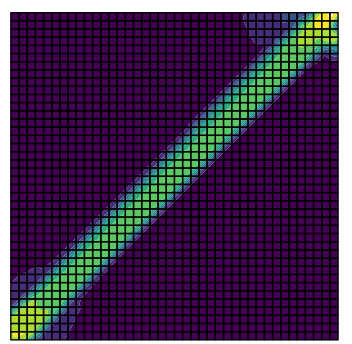

(k)
Non-Local G\&S

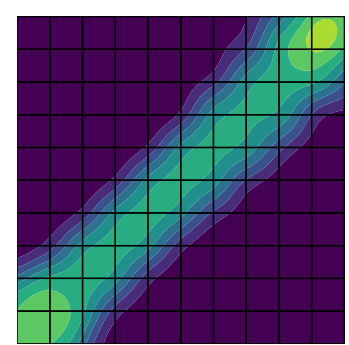

(c)

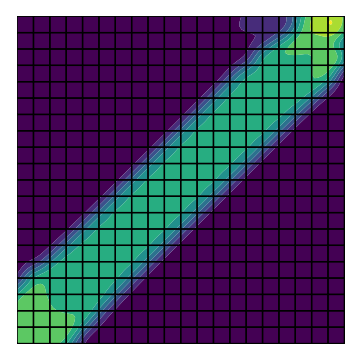

(f)

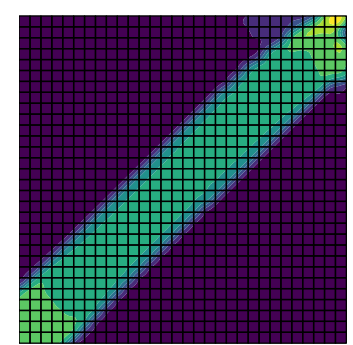

(i)

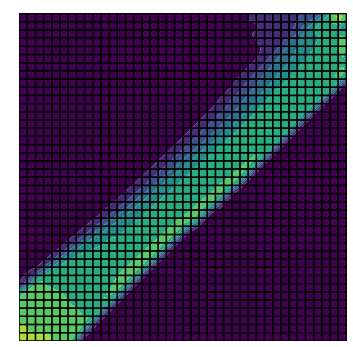

(1)

Intact

Remoulded

Degree of Remoulding

Figure 5: Sensitivity distribution at maximum compression for (a,b,c) $10 \times 10,(d, e, f) 20$ x 20, (g,h,i) $30 \times 30$ and $(\mathrm{j}, \mathrm{k}, \mathrm{l}) 40$ x 40 element discretisations for $(\mathrm{a}, \mathrm{d}, \mathrm{g}, \mathrm{j})$ local undrained $(\mathrm{b}, \mathrm{e}, \mathrm{h}, \mathrm{k})$ non-local (Gaussian) undrained; and $(\mathrm{c}, \mathrm{f}, \mathrm{i}, \mathrm{l})$ non-local $(\mathrm{G} \& \mathrm{~S})$ undrained smallstrain analyses. 


\subsection{Influence of characteristic length and softening scaling}

In the non-local strain-softening method, the characteristic length $l_{c}$ controls the extent of the strain localisation and thus, indirectly, the strainsoftening rate. Figure 6 presents the responses obtained from the analyses with 20x20 mesh (i.e. 400 elements) using Gaussian and G\&S methods with three different values of $l_{c}$. Figure 7 depicts the degree of the soil remoulding at the maximum compression of the mesh. In Figures $6 \mathrm{a}$ and $\mathrm{b}$, as $l_{c}$ increases the effective area over which the non-local averaging is performed increases, and therefore, the strain softening rate decreases (compare Fig $7 \mathrm{a}$ with $\mathrm{b}$ and $7 \mathrm{c}$ with $\mathrm{d}$ ). For the same $l_{c}$ value, the size of strain-softened area is slightly larger in the case of the G\&S method in comparison to the Gaussian method, due to the wider distribution of G\&S weighting function (see Figure 1). Therefore, a non-local softening model based on a critical state model has three principle parameters controlling the strain-softening rate: $k, A$ and $l_{c}$.

The simulation of strain-softening in practical geotechnical engineering boundary value problems necessitates the use of a very small value of $l_{c}$ if the scale of shear band is to be modelled at true scale (i.e. of the order of, at most, a few millimetres for the fine-grained soils; Gylland et al. [44], and 10-20 $D_{50}$ for sands; Mühlhaus and Vardoulakis [3] and Muir Wood [45]). However, the non-local averaging is only effective when $l_{c}$ is larger than the size of the smallest element in the FE mesh, which necessitates ultra fine domain discretisations rendering such analyses computationally impractical. In order to overcome this problem, a softening scaling rule was suggested in the literature $[46,29,47,25]$, which postulates that a much larger thickness of shear band can be adopted to describe the correct load-displacement response in a numerical model, provided that: (i) the softening rate is adjusted accordingly to compensate; and (ii) the mechanism is not likely to be significantly altered by such scaling. Advanced soil deformation measurement techniques, such as Particle Image Velocimetry, must be applied in geotechnical physical model tests to identify these constraints on mechanism. A linear relationship between the strain-softening rate and the characteristic length is generally shown to be effective $[25,27]$. To investigate the effect of the softening scaling, two sets of analyses were performed with the non-local G\&S method considering a scaling ratio (i.e. $k / l_{c}$ ) of four. In the first set, the same analysis mesh was used whilst the parameters $k$ and $l_{c}$ were varied, while both mesh and parameters $k$ and $l_{c}$ were varied in the second set of analyses. The load-displacement responses obtained from these analyses are 
presented in Figure 8. The force-displacement responses are almost identical for both sets of analyses, demonstrating that the linear softening scaling rule is also generally valid for the non-local SMCC constitutive model. Figure 9 plots the contours of the degree of soil remoulding for selected cases from both sets of analyses and illustrates the following points:

1. Identical meshes with different $l_{c}$ (c.f. Figure $9 \mathrm{a}$ and $9 \mathrm{c}$ where $l_{c}=0.05 \mathrm{~m}$ and $0.10 \mathrm{~m}$, respectively) demonstrate that the size of strain-softened zone is different and proportional to the value of $l_{c}$, yet practically identical load-displacement responses are obtained (see Figure 8a).

2. Different meshes with different $l_{c}$ (c.f. Figure $9 \mathrm{~b}$ and Figure $9 \mathrm{~d}$ where $l_{c}=0.05 \mathrm{~m}$ and $0.025 \mathrm{~m}$, respectively) reveal that the size of strainsoftened zone is proportional to the value of $l_{c}$ and yet practically identical load-displacement responses are obtained because the ratio $k / l_{c}$ is constant (see Figure 8b).

3. Different mesh with identical $l_{c}$ (c.f. Figure 9a and Figure 9b where $l_{c}=0.05 \mathrm{~m}$ ) show, as demonstrated earlier in Figure 5, that the size of strain-softened zone has been regularised by the addition of the characteristic length in the strain averaging procedure.

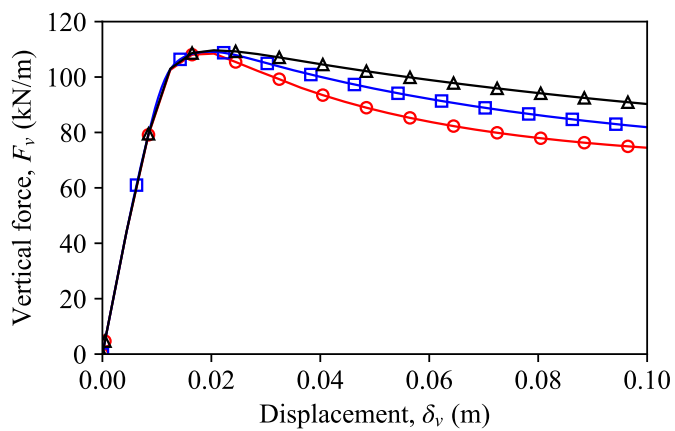

(a)

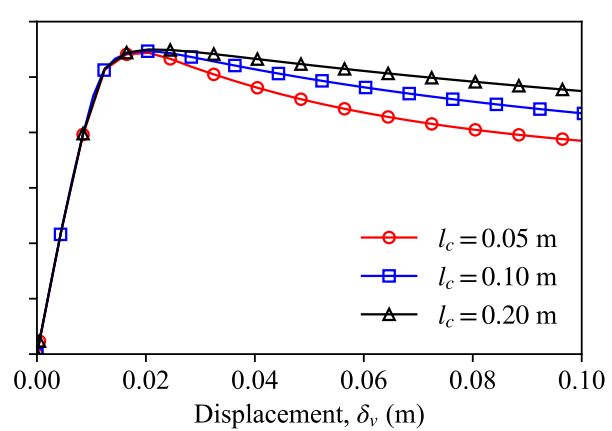

(b)

Figure 6: Non-local small-strain simulation response for varying characteristic length (20 x 20 elements): (a) non-local (Gaussian) undrained; and (b) non-local (G\&S) undrained. 


$$
l_{c}=0.05 \mathrm{~m}
$$

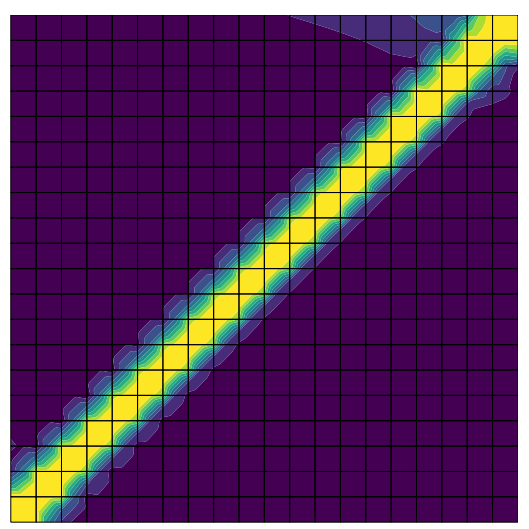

(a)

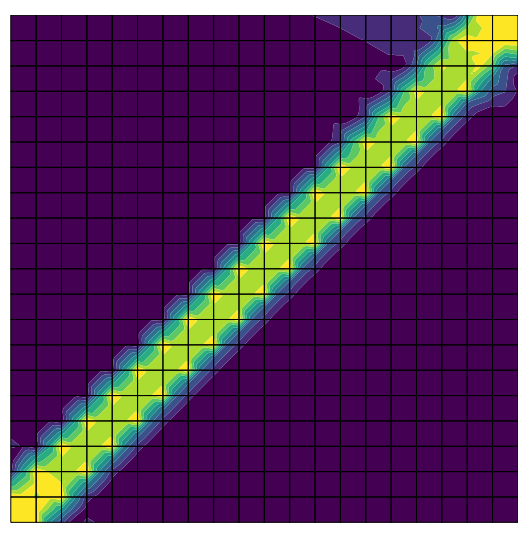

(c)

$$
l_{c}=0.20 \mathrm{~m}
$$

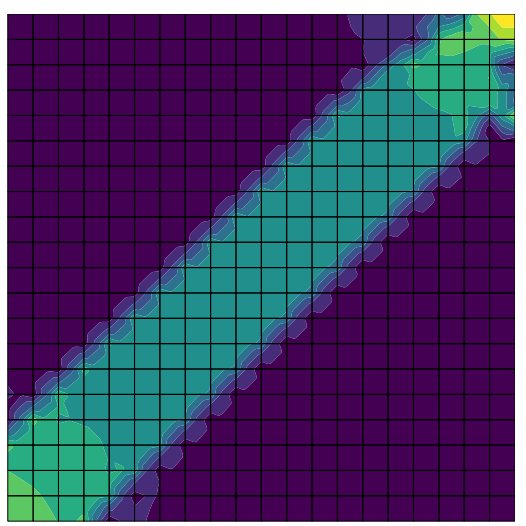

(b)

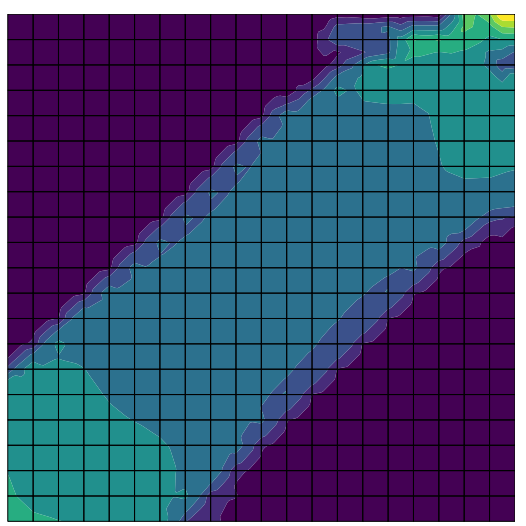

(d)

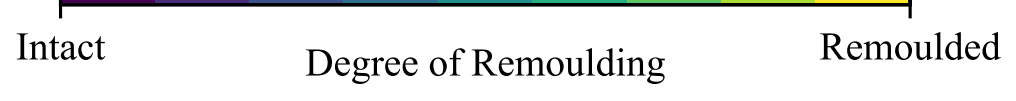

Figure 7: Influence of varying characteristic length on Sensitivity distribution at maximum compression: (a) non-local (Gaussian) undrained with $l_{c}=0.05 \mathrm{~m}$; (b) non-local (Gaussian) undrained with $l_{c}=0.20 \mathrm{~m}$; (c) non-local $(\mathrm{G} \& \mathrm{~S})$ undrained with $l_{c}=0.05 \mathrm{~m}$; and (d) nonlocal (G\&S) undrained with $l_{c}=0.20 \mathrm{~m}$. 


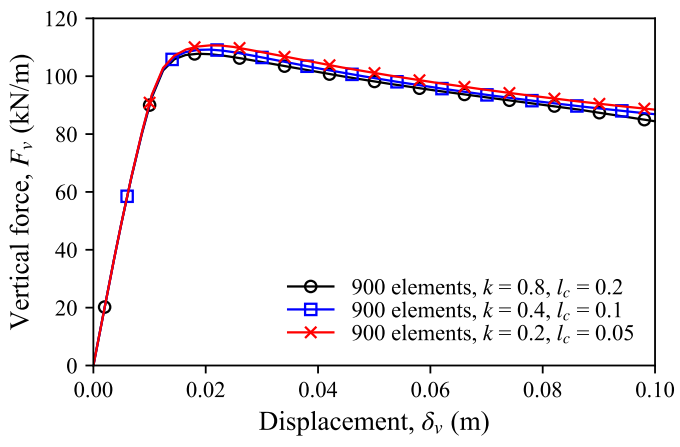

(a)

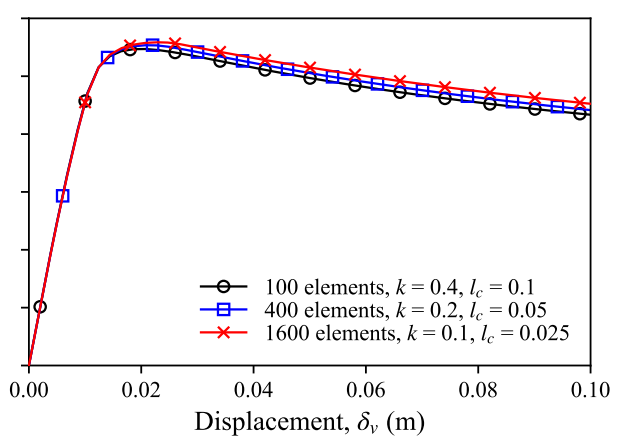

(b)

Figure 8: Influence of softening scaling with $k / l_{c}=4$ for : (a) same mesh with varying $k$ and $l_{c}$; and (b) different mesh with varying $k$ and $l_{c}$. 
$l_{c}=0.05 \mathrm{~m}$

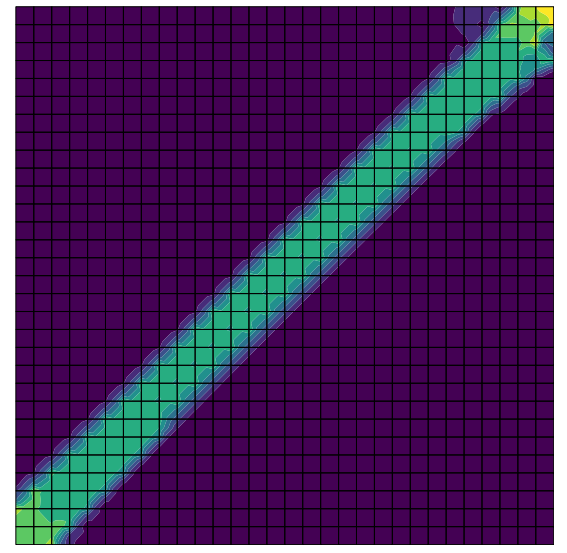

$l_{c}=0.10 \mathrm{~m}$

(a)

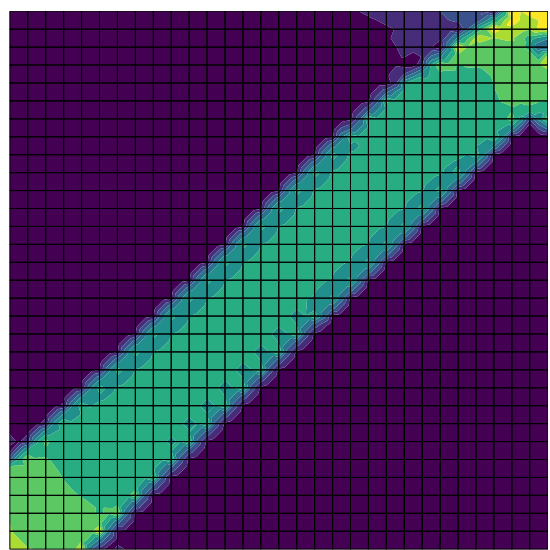

(c) $l_{c}=0.05 \mathrm{~m}$

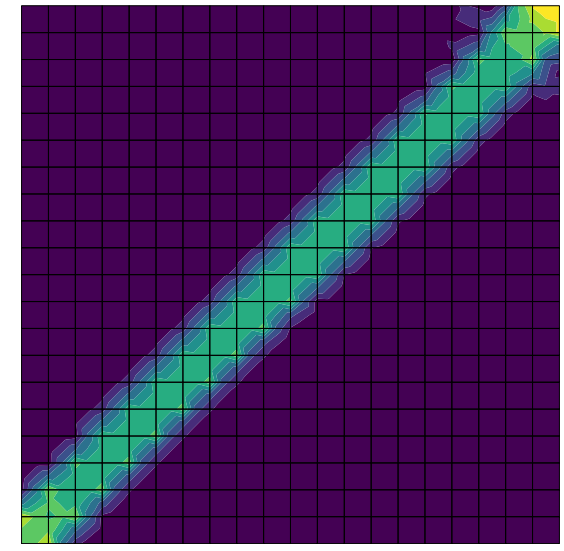

$l_{c}=0.025 \mathrm{~m} \quad$ (b)

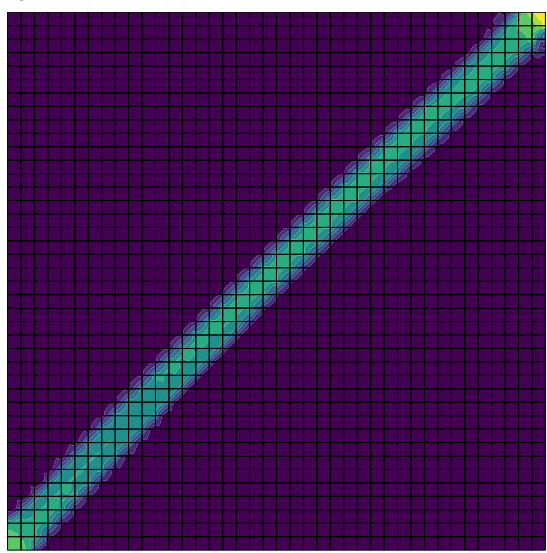

(d)

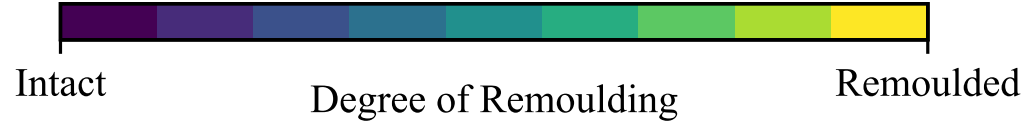

Figure 9: Influence of softening scaling on the size of softening zone: (a) 900 elements, $k=0.20, l_{c}=0.05 \mathrm{~m}$; (b) 400 elements, $k=0.20, l_{c}=0.05 \mathrm{~m}$; (c) 900 elements, $k=0.40, l_{c}=0.10$ $\mathrm{m}$; and (d) 1600 elements, $k=0.10, l_{c}=0.025 \mathrm{~m}$. 


\section{Simulation of a classical large deformation boundary value prob- lem: buried pipe uplift}

Buried pipelines transport hydrocarbons at elevated temperatures and pressures. The elevated temperatures and pressures can cause upheaval buckling of the pipe in the direction of the least soil resistance. The process of pipe uplift buckling is a large deformation problem in which significant pipe movements and soil deformations are involved. In this section, the mesh dependency of local and non-local strain-softening models when implemented in FE simulation of the pipe uplift process is investigated.

\subsection{Modelling details}

Large deformation fully-coupled effective stress finite element analyses were performed using plane strain boundary conditions in Abaqus. The LDFE approach adopted in this paper is based on the Remeshing and Interpolation Technique with Small Strain (RITSS) strategy [21], where a large deformation finite element analysis is divided into several small strain finite element analyses in order to alleviate issues related to mesh distortion. A new mesh is generated at the beginning of every small strain step based on the deformed geometry of the previous step, and the stresses, field variables and state variables are then mapped to the new mesh from the old deformed mesh. The implementation of the RITSS technique into the Abaqus software package has been described in detail previously by Wang et al. [48], Chatterjee [37], and Tian et al. [49].

A two dimensional plane strain model was developed assuming a 'wishedin-place' pipe with a diameter $D$ of $0.5 \mathrm{~m}$ and an initial embedment $H$ of $4 D$. The pipe was modelled as a solid rigid part and the soil was modelled as a solid deformable part. The interaction between the pipe surface and the soil was assumed to be rough and fully bonded. Four mesh densities (coarse, medium, fine and very fine) of eight-noded plane strain quadrilateral, biquadratic displacement, bi-linear pore pressure, reduced integration elements (CPE8RP in the Abaqus Standard library) were selected to discretise the soil. A square partition zone with a size of $3 D$ by $3 D$ containing smaller elements was created around the pipe to optimise the analysis mesh. The adopted mesh discretisations are illustrated in Figure 10. Displacement controlled uplift analyses were performed using the local SMCC model and non-local G\&S method. To perform the non-local spatial averaging, a characteristic length of $0.1 \mathrm{~m}$ (equal to the mesh element size at the partition edge of 
the coarse mesh) is assumed in all the non-local softening calculations. To establish initial normally consolidated conditions, a surcharge pressure of 10 $\mathrm{kPa}$ was applied at the top boundary of the mesh. The pipe was present when the surcharge pressure was applied, and the 'correct' initial vertical and horizontal stresses were imposed directly throughout the soil domain. The value of lateral coefficient of earth pressure at rest, $K_{o}$, was considered equal to $1-\sin \left(\phi_{c}\right)$, where $\phi_{c}$ is the critical state effective friction angle of the soil. The SMCC model parameters for the Pisa clay were once again adopted, the permeability of the soil was assumed as $10^{-9} \mathrm{~m} / \mathrm{sec}$, and the pipe was displaced vertically by $0.5 D$ in 50 RITSS steps.

\subsection{Results: mesh dependency}

Figure 11 shows the numerical results for the undrained uplift of the pipe using local and non-local G\&S methods for different meshes. The uplift resistance normalised by the diameter and intact shear strength at the level of the pipe centre, $V_{u} / s_{u i} D$, (calculated from equation 15) is plotted against normalised pipe displacement $\left(d_{\text {pipe }} / D\right)$. Assuming negligible softening occurs until the intact undrained shear strength is mobilised, the shear strength of NC clay predicted by the SMCC model under the plane strain conditions is calculated as:

$$
s_{u i}=\frac{2}{\sqrt{3}} \frac{M}{2} \exp \left[\frac{\left(\lambda^{*}-\kappa^{*}\right) \ln \left(p_{c i} s_{e p i} / 2\right)+\kappa^{*} \ln \left(p_{i}^{\prime}\right)}{\lambda^{*}}\right]
$$

where $p_{c i}$ is the initial value of the quantity $p_{c}$ and $p_{i}^{\prime}$ is the initial mean effective stress.

The upper bound derived by Randolph and Houlsby [50] for uniform Tresca soil and a fully bonded contact condition of 11.94, and responses from elastic-perfectly plastic FE analyses by Maitra et al. [51], are also plotted alongside for comparison purposes. The curve for the case with no strainsoftening matches with the published results well. The mesh dependency of the uplift response is clearly observed for the local undrained analyses in Figure 11a. The refinement of the mesh from coarse to very fine causes gradual increase in the strain-softening rate and leads to a decrease in the peak resistance. The peak uplift capacities for the simulations with fine and very fine mesh density are also slightly lower than the corresponding values for cases with the coarse and medium mesh density. As expected, the non-local analyses demonstrates significantly reduced mesh dependency (see Figure 11b) with all the curves falling in a narrow band. 


\subsection{Computational costs}

Non-local averaging is often considered to be a computationally expensive process in the FE simulations; however, this is potentially a misconception. Figure 12 presents a bar chart in which the relative computational time of the local and non-local G\&S undrained analyses $\left(t_{a} / t_{a, r e f}\right)$ is plotted for the analyses with the four different meshes. The computational time for the nonlocal analysis with very fine mesh is taken as the reference time $\left(t_{a, r e f}\right)$. In only half of the cases was the non-local analysis slower than the corresponding local analysis. The maximum increase in computational time $(\approx 16 \%)$ between the local and non-local analyses was for the case with the very fine mesh. The non-local analysis with the fine mesh density was even $\approx 30 \%$ faster than the corresponding local analysis. This shows that the application of non-local methodologies to ultra large deformation geotechnical analyses is possible with negligible additional computational burden. 


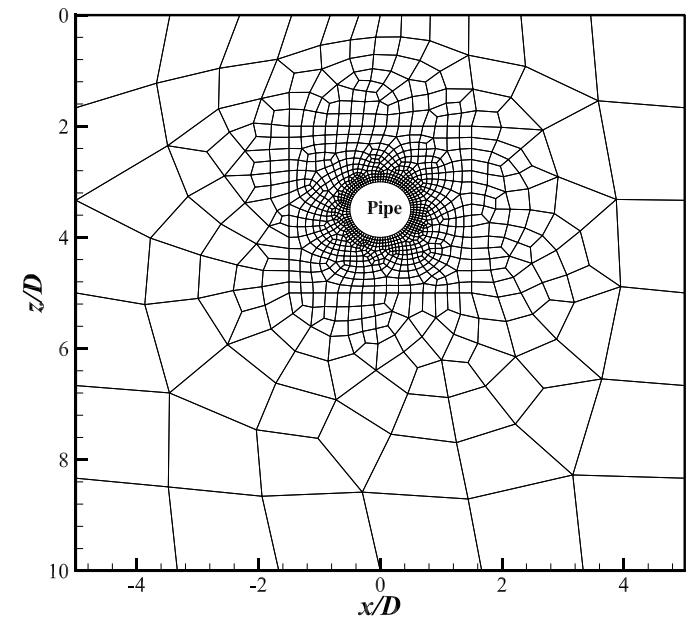

(a)

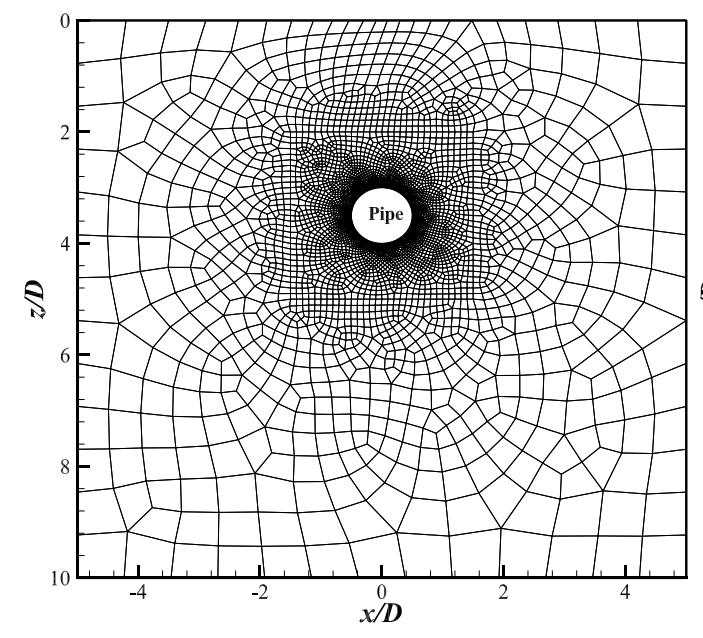

(c)

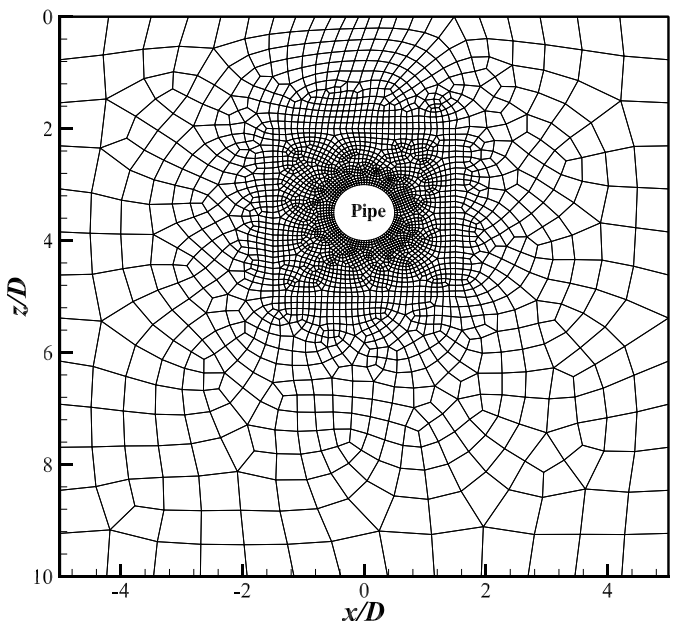

(b)

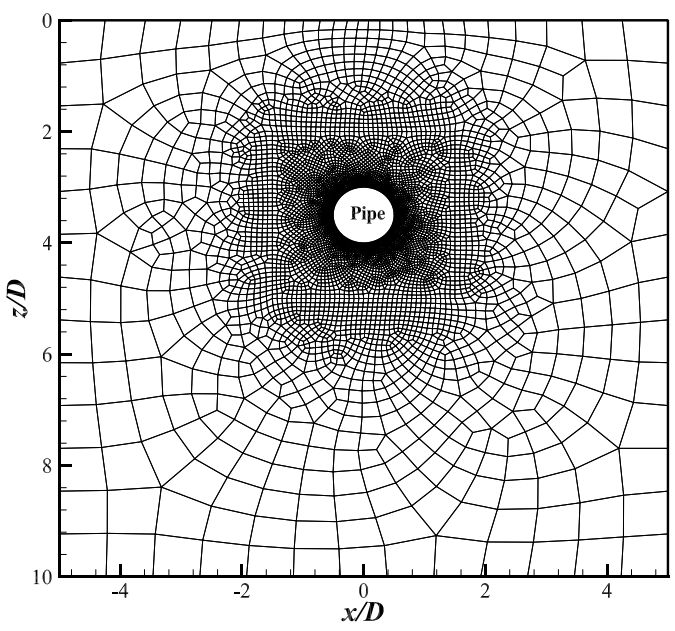

(d)

Figure 10: Illustration of the initial analysis mesh used for the buried pipe simulations: (a) coarse; (b) medium; (c) fine; and (d) very fine. 


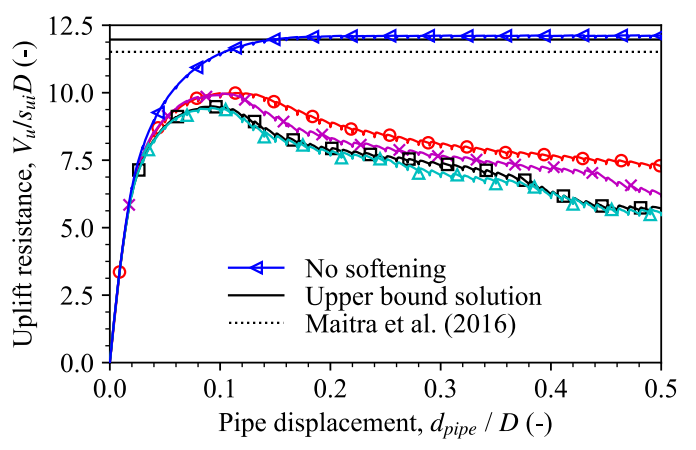

(a)

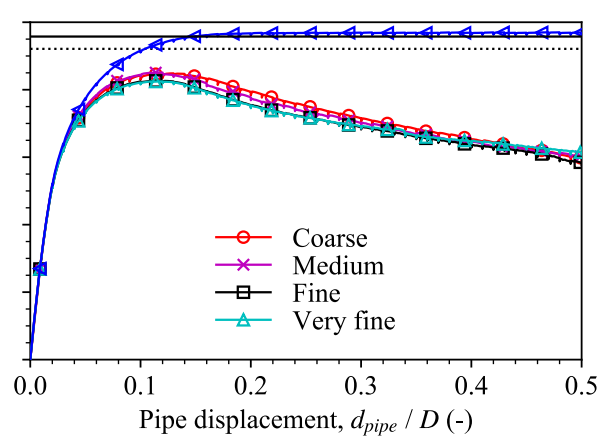

(b)

Figure 11: Large deformation simulation of buried pipe uplift response for varying mesh density: (a) local undrained; and (b) non-local (G\&S) undrained.

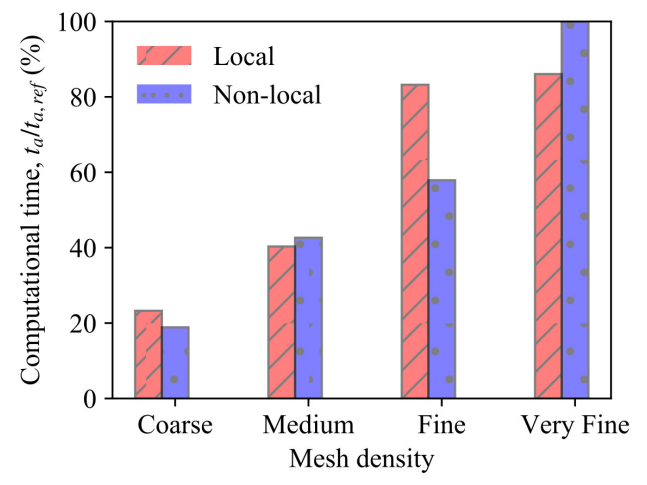

Figure 12: Comparison of total analysis time for large deformation simulation of buried pipe undrained uplift response with local and non-local G\&S softening models for varying mesh density.

\section{Simulation of cyclic T-bar penetration}

The T-bar penetrometer is a popular offshore geotechnical site characterisation tool for profiling soft clay soils. The T-bar is passed through the soil at an undrained rate $\left(v D / c_{V}>10\right.$ where $v$ is penetration rate and $c_{V}$ is coefficient of consolidation; e.g., Lehane et al. [52] and Colreavy et al. [53]) and the measured penetration resistance is converted to an interpreted soil strength using plasticity solutions [50]. A cyclic T-bar penetration test, where the T-bar is moved up and down repeatedly over a distance of a few diameters, allows evaluation of the intact and remoulded soil strengths as 
well as the rate of strength degradation. This genera of test is potentially far better suited to deriving the soil sensitivity $S_{t}$ and the strain-softening rate parameter $k$ because a fully remoulded state is reached. This allows the sensitivity $S_{t}$ to be unambiguously defined, which in turn reduces the uncertainty associated with determining an appropriate value of $k$. Further to that, if the T-bar is left in place for some time after the end of this cycling (allowing dissipation of the excess pore pressures previously generated), the consolidated-undrained soil strength can also be assessed by performing another episode of undrained cycles [54].

The penetration resistance in soft clay soils depends on both the strain rate and the consolidation characteristics of the soil as shown by Lehane et al. [52]. In this paper, the non-local SMCC model, which is a rate-independent model and thus ignores the strain-rate effects on the T-bar resistance, is applied to simulate a cyclic T-bar penetration test using the RITSS technique. It is assumed that during an undrained analysis, no dissipation of the generated excess pore pressure occurs. Firstly, an undrained episode comprising five penetration cycles was performed using the local and non-local model variants, demonstrating important characteristics of the non-local approach in LDFE analyses. Secondly, analyses including an episode of undrained penetration, subsequent consolidation analysis, followed by another episode involving one cycle of the consolidated-undrained penetration were simulated.

\subsection{LDFE modelling details}

A $D=0.04 \mathrm{~m}$ diameter T-bar was modelled under plane strain boundary conditions, with the FE analysis mesh shown in Figure 13. Due to the symmetrical geometry of the problem, only half of the domain was modelled for computational efficiency. The T-bar was 'wished-in-place' with an initial embedment of $5.5 \mathrm{D}$ and the width and height of the soil domain were taken as $10 D$ and $15 D$, respectively. Following Zhou and Randolph [14], in order to minimise the propagation of interpolation errors in the stress state and state variables caused by repeated remeshing, a partial remeshing scheme was used where only the region close to the T-bar was periodically remeshed. The remeshing region comprises very fine elements near to the T-bar and then gradually transitions to a coarse mesh in the non-remeshed far field (see Figure 13). The T-bar-soil surface interaction was considered to be smooth with normal separation not allowed (i.e. no breakaway).

To obtain the cyclic penetration response, displacement controlled analyses were performed. The T-bar was penetrated by $4 D$ from the initial 
position in the first penetration cycle to partially remould the soil, establishing an approximately steady value of normalised resistance, $V_{u} / s_{u i} D$, following the initial peak. Penetration cycles with $3 D$ amplitude were then carried out until the fully remoulded asymptotic state was achieved. The mesh discretisation was performed using eight-noded biquadratic plane strain quadrilateral, reduced integration elements (CPE8R in the Abaqus Standard library) for the undrained penetration cycles. To perform consolidation analyses, eight-noded plane strain quadrilateral, biquadratic displacement, bilinear pore pressure, reduced integration elements (CPE8RP in the Abaqus Standard library) were chosen to discretise the soil domain. The undrained stress-strain soil response was described by adding the bulk modulus of the pore water to the effective stress-strain matrix in an Abaqus 'static' step. The soil state at the end of undrained cycles was mapped to a coupled porefluid effective stress analysis (an Abaqus 'soils' step) to allow the dissipation of the excess pore pressures. The undrained cycles following the consolidation step were again considered as a 'static' step by augmenting the effective stress-strain matrix once again with the bulk modulus of water. This approach resulted in improved convergence compared to a purely fully-coupled analysis approach.

The SMCC model parameters for the UWA kaolin clay are provided in Table 1. For non-local spatial averaging calculations, a characteristic length $l_{c}$ value of $0.01 \mathrm{~m}$ was selected to ensure the non-local calculations are effective in the very fine mesh region, which captures the failure mechanism mobilised by T-bar penetration. Matching of the simulation response to the T-bar experiment on a cycle-by-cycle basis will result in a set of strain-softening parameters relevant only to this characteristic length. However, parameter scaling as described earlier [25] could be used to scale the parameters for a different characteristic length for use in a problem with a different scale (whether larger or smaller). Similar to the pipe analyses, the initial normally consolidated state in the soil domain was achieved by applying a surcharge pressure of $50 \mathrm{kPa}$ at the top boundary of the mesh and the 'correct' vertical and horizontal stresses were imposed as an initial stress condition throughout the soil domain. The value of lateral coefficient of earth pressure at rest, $K_{o}$, was considered equal to $1-\sin \left(\phi_{c}\right)$, where $\phi_{c}$ is the critical state effective friction angle of the soil. The permeability of the soil was taken as $10^{-9} \mathrm{~m} / \mathrm{sec}$. 


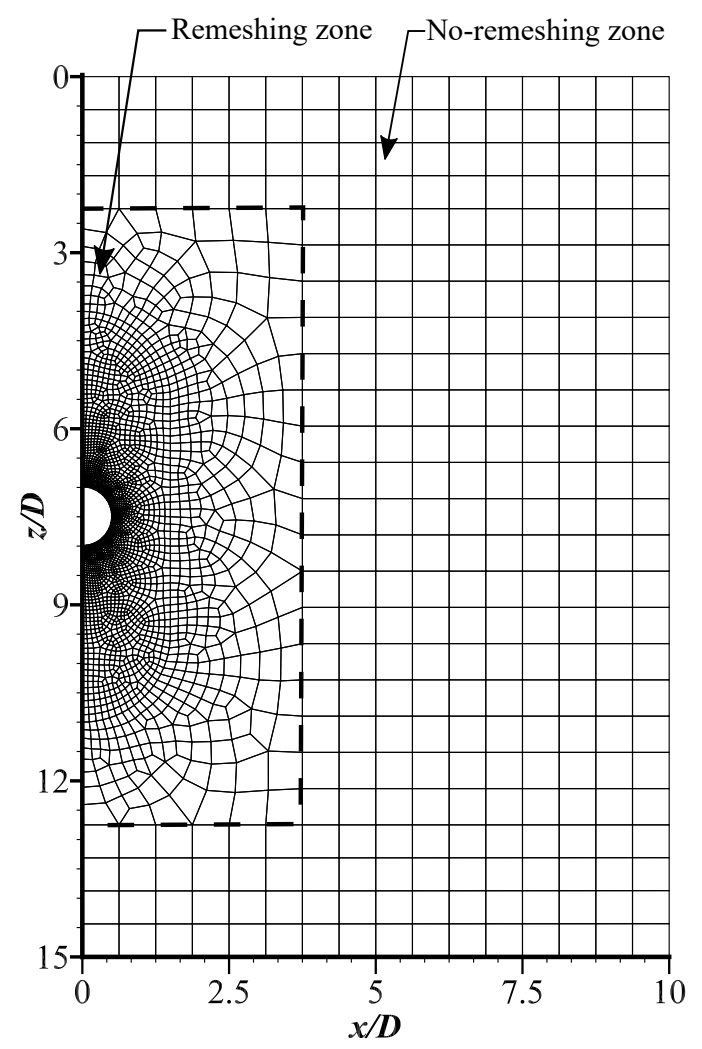

Figure 13: Illustration of the FE analysis mesh used for the cyclic T-bar simulations.

\subsection{Results: undrained and consolidated-undrained penetration}

The results obtained from numerical simulations of the undrained T-bar cycles using the local and non-local G\&S softening methods are plotted in Figure 14. Undrained T-bar penetration resistance normalised by the diameter and intact shear strength at the level of the T-bar centre, $V_{u} / s_{u i} D$, (calculated from equation 15) is plotted against normalised displacement $\left(d_{T-\text { bar }} / D\right)$ in Figures 14a and b. Figures $14 \mathrm{c}$ and d show numerically obtained penetration resistance degradation factor $\left(V_{u} / V_{u i}\right)$ at the mid-depth of each T-bar pass (i.e. $d_{T-b a r} / D$ of 2.5) plotted against the penetration cycle number $(N)$, following the convention suggested by Randolph et al. [17] and Zhou and Randolph [14] that the first pass is referred to as cycle number $N=0.25$. The results extracted from five cyclic undrained T-bar penetration tests in UWA kaolin clay by Ragni et al. [35] and O'Loughlin et al. [55] are also presented in the figure with error bars representing the 
range and the symbols representing the mean value for the five tests. The contour plots of the local incremental equivalent plastic strain variable, $\dot{\varepsilon}_{s}$, are presented in Figure 15 for the points A-H marked in Figure 14.

Similar to the pipe uplift simulations, the normalised resistance first mobilises a peak value before decreasing rapidly to a steady value with further advancement for both local and non-local analyses. The initial resistance profile in the local simulation shows oscillations, but these are significantly reduced in the non-local analysis (c.f. points A, B and C in Figure 14a with points E, F and $\mathrm{G}$ in Figure 14b). Figure 14c and $\mathrm{d}$ demonstrate that the numerically obtained $V_{u} / V_{u i}$ values at the residual state are higher than those derived from the experiments. In the analysis this is caused by: (i) partial remoulding of the soil caused by the first pass of the T-bar, first reported by Zhou and Randolph [14]; and (ii) over-estimation of the strain-softening rate parameter $k$ resulting in a lower penetration force $\left(V_{u i}\right)$ during the first pass, by which all other measurements are normalised. The oscillations observed in the resistance profile of the local analyses are expected in non-regularised strain-softening materials including clays and are caused by periodic development and diffusion of localised shear bands (c.f. Figures $15 \mathrm{a}, \mathrm{b}$ and c), which was also highlighted by Zhou and Randolph [56]. When the soil is partially remoulded the oscillations disappear because the mechanisms stabilises, with a unique pattern of plastic deformation formed as observed in Figure 15d at the point D, which is chosen at the same level as point A in the third pass of the T-bar penetration. Such artefacts are less evident in the non-local strain-softening simulations because the strain-localisation is adequately regularised and a practically identical zone of plastic deformation is formed after any level of further deformation (at points E, F and G in the first $\mathrm{T}$-bar pass and point $\mathrm{H}$ in the third T-bar pass) (c.f Figures 15e, f, g and $h$ ).

Several additional analyses were carried out to back-calculate the values of the softening parameters (i.e. $k$ and $S_{t}$ ) that lead to a response comparable with that observed in the experiment. The values yielding the 'best fit' presented in Figure 16 (excess pore pressure contours) and Figure 17 (penetration resistance response) are $k=0.06$ and $S_{t}=2.78$, which is a decrease in strain-softening rate by a factor of 3.33 and an increase in sensitivity by a factor of 1.26. The question is: could these parameters have been assessed directly from the T-bar penetration test data? 


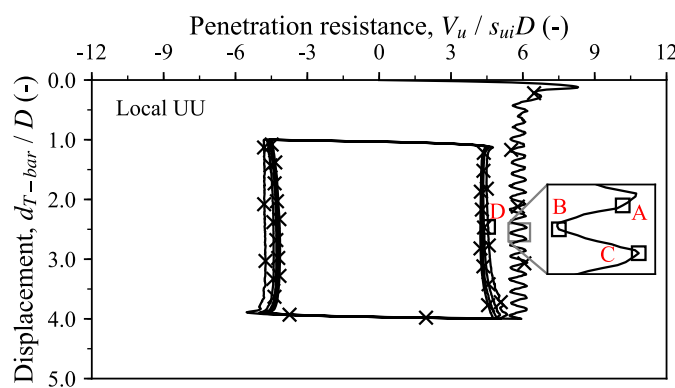

(a)

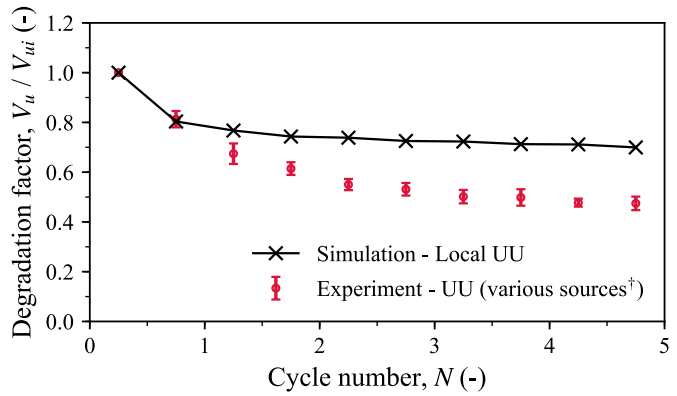

(c)

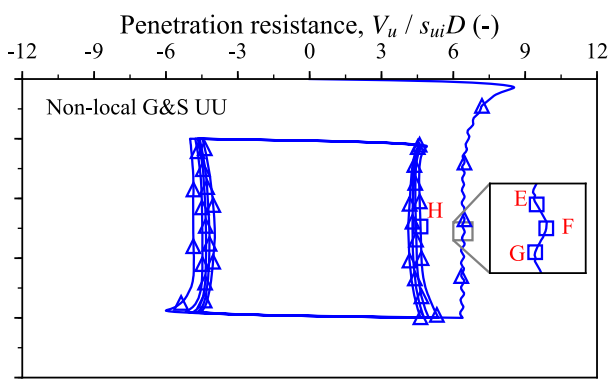

(b)

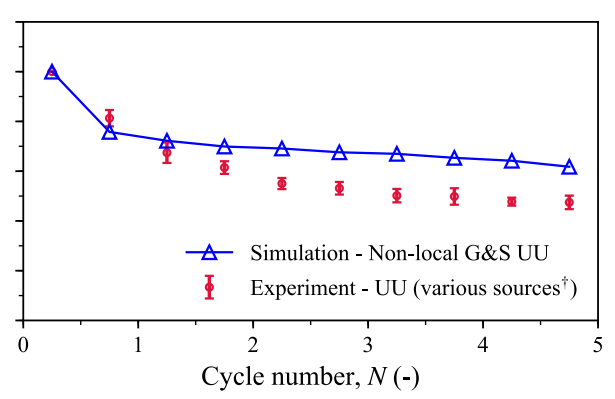

(d)

${ }^{\dagger}$ Ragni et el. 2015; O’Loughlin et al. 2020

Figure 14: Large deformation local and non-local simulations of cyclic undrained T-bar penetration with $k=0.2$ and $S_{t}=2.2$. 

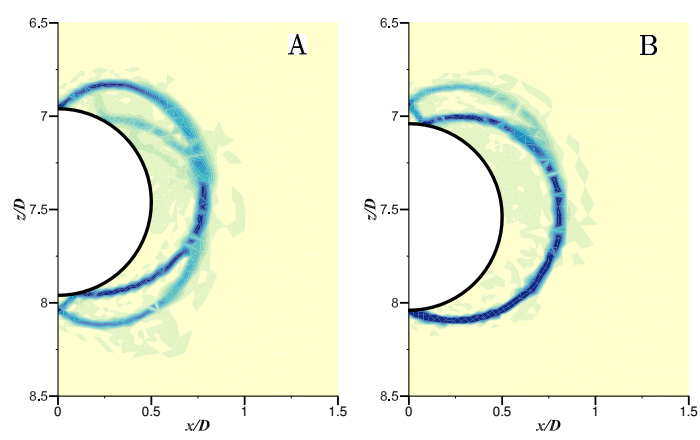

(a)

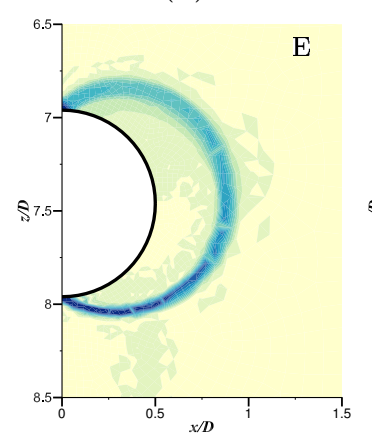

(e)

(b)

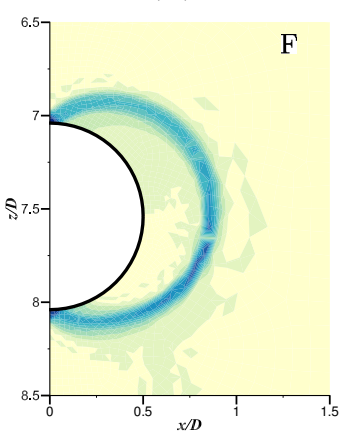

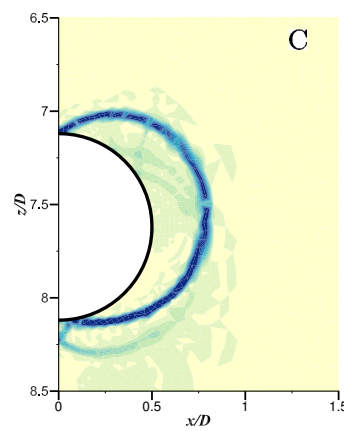

(c)
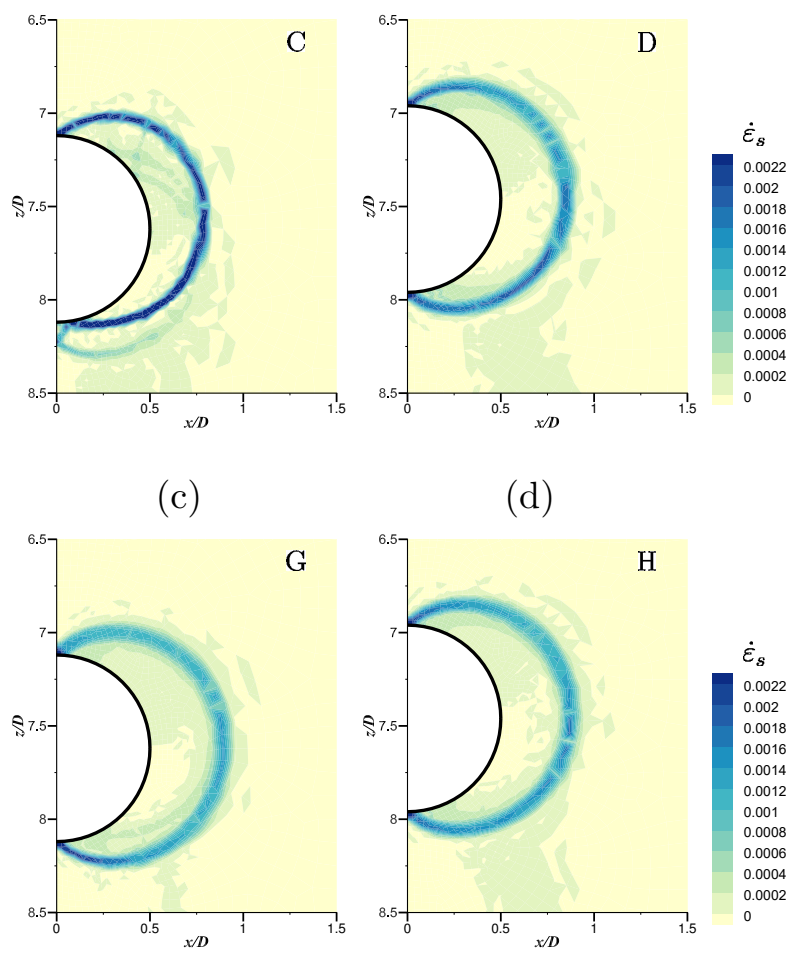

(d)

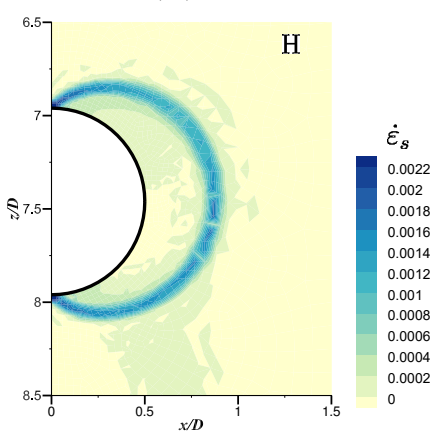

(g)

(h)

Figure 15: Evolution of the distribution of the incremental local equivalent plastic strains $\left(\dot{\varepsilon}_{s}\right)$ in the first and third T-bar pass: (a, b, c and d) local analyses, and (e, f, g and h) non-local analyses. 
Rather than use element test data to define strain-softening parameters, Einav and Randolph [10] suggested a method of extracting the degradation parameters $\xi_{s, 95}$, which is an equivalent general shear strain required to cause $95 \%$ strength degradation, and the sensitivity $S_{t}$ from cyclic full-flow penetrometer tests. Zhou and Randolph [14] proposed the following equation to estimate $S_{t}$ from T-bar test based sensitivity $S_{T-b a r}$ :

$$
S_{t}=\left[1+0 \cdot 3(1-\alpha)^{1 \cdot 5}\right]\left[\delta_{\mathrm{rem}}^{\prime \prime}+\left(1-\delta_{\mathrm{rem}}^{\prime \prime}\right) \mathrm{e}^{1 \cdot 5 \xi / \xi_{s, 95}}\right] S_{T-b a r}
$$

in which the parameter $\xi$ is the current accumulated general shear strain, $\delta_{\text {rem }}^{\prime \prime}$ represents the disturbance during initial penetration and $\alpha$ is the inverse of the initial sensitivity (i.e. $1 / S_{t}$ ). According to Einav and Randolph [10], $\xi_{s, 95}$ can be obtained from:

$$
\xi_{s, 95}=2 \xi_{\mathrm{P}} N_{95}
$$

where $\xi_{\mathrm{P}}$ was obtained from the Upper Bound Strain Path Method (UBSPM) for a smooth T-bar as 4.42, and $N_{95}$ is the number of cycles required for $95 \%$ strength degradation to occur in a full-flow penetrometer test. Based on their numerical analyses, Zhou and Randolph [14] obtained values for $N_{95}$ in the range of 3.0-4.0, depending upon the sensitivity of the soil assumed. From the cyclic T-bar penetration test data (see Figure 17b), the $N_{95}$ value appears to be around 2.0 for kaolin clay. This results in a value of $\xi_{s, 95}$ of 17.68.

To relate $k$ in the $\mathrm{SMCC}$ constitutive model to this value of $\xi_{s, 95}$, we begin by integrating Equation 6, which yields:

$$
s_{e p}=s_{e p f}+\left(s_{e p i}-s_{e p f}\right) \exp \left[-\frac{k}{\lambda^{*}-\kappa^{*}} \varepsilon_{s}\right]
$$

in which the parameter $\varepsilon_{s}$ represents the average magnitude of plastic shear strain operative in the full flow mechanism during one T-bar penetration/extraction stroke.

For the undrained T-bar penetration, if it is assumed that $\dot{\varepsilon}_{v}^{\mathrm{p}}<<\dot{\varepsilon}_{q}^{\mathrm{p}}$, then Equation 18 reduces to:

$$
s_{e p}=s_{e p f}+\left(s_{e p i}-s_{e p f}\right) \exp \left[-\frac{k \sqrt{A}}{\lambda^{*}-\kappa^{*}}\left(\varepsilon_{q}^{\mathrm{p}}\right)\right]
$$


By combining Equation 19 with the generalised form of the Einav-Randolph strength degradation law [10], we generate the following relation between $k$ and $\xi_{s, 95}$ :

$$
k=\frac{3\left(\lambda^{*}-\kappa^{*}\right)}{\xi_{s, 95} \sqrt{A}}
$$

The values of the parameters $\lambda^{*}$ and $\kappa^{*}$ used in the T-bar penetration analyses were 0.0811 and 0.019 respectively. For the softening rate, $k$ derived from the element tests, i.e. $k=0.2$, the $\xi_{s, 95}$ value from equation 20 is 2.08 which is much lower than suggested range of around 10 to 50 [10]. This is at least partly because the strain-softening rate parameter $k$ was calibrated from standard triaxial tests, which are ill suited because: (i) they do not impart sufficient deformation for the soil to reach a residual state [45]; and (ii) the strain-localisation that typically occurs within a triaxial sample leads to erroneous over-estimation of the strain-softening rate because the strain-rate applied at the boundaries of the sample is far lower than the operative strain-rate mobilised within the strain-localisation within the sample. Therefore, $\xi_{s, 95}$ of 17.68 from equation 17 would imply that a value of $k$ of 0.01 ought to yield the correct rate of strain-softening. However, this is six times lower than the 'best-fit' value of 0.06 derived by trial and error. In other words, a much faster than expected rate of strain-softening was required for the simulation to fit the experimental measurements. One reason for this is that the non-local softening method attenuates the strain-softening rate with increasing characteristic length. For this numerical simulation, the attenuation was around $11 \%$ for the $l_{c}$ value selected in this paper (compare Figures 14a and b). For a larger characteristic length this attenuation would be even more significant, therefore, as illustrated also for the pipe uplift problem, the strain-softening parameters are intrinsically linked to the choice of characteristic length. This demonstrates that the UBSPM method proposed by Einav and Randolph [10] is - like element tests - similarly unable to determine appropriate strain-softening parameters, such as $\xi_{s, 95}$ and hence $S_{t}$ (or $k$ and hence $S_{t}$ for a critical state based constitutive model) directly from cyclic T-bar penetration test data for simulations that utilise non-local regularisation techniques to mitigate mesh dependency. This limitation of the UBSPM method was also highlighted by Einav and Randolph [10], and is due to existence of a velocity discontinuity in the kinematic mechanism for the cylinder-based problems - such as the T-bar penetration - leading to 
a finite jump in the cumulative shear strain when a soil element traverses a discontinuity. New methods of calibration are required if any non-locally regularised strain-softening models are to be of practical use.

The first two penetration and extraction strokes of the undrained episode with parameters $k=0.06$ and $S_{t}=2.78$ were also repeated using the effective stress approach coupled with pore pressure in order to compare the predicted excess pore pressures with those obtained using a fully undrained approach. Figure 16 presents contours of excess pore pressures $u_{\text {excess }}$ using: (i) normal elements (CPE8R in the Abaqus standard library) with bulk modulus equal to that of the pore water (Figures 16a and c); and (ii) pore pressures elements (CPE8RP in the Abaqus standard library) with pore pressures calculated using Biot's equations (Figures 16b and d), after T-bar advancement of $0.3 D$ in the first penetration pass computed (Figures 16a and b) and in the first extraction pass at the same advancement from the bottom of the penetration pass (Figures 16c and d). The analysis with pore pressure elements was performed with a normalised penetration velocity, $v D / c_{v}$, of 100 . The distributions of the excess pore pressures obtained using the two approaches are practically identical for the first penetration and extraction passes. In the T-bar penetration pass, the suction pressures develop behind the T-bar because of the extension loading, and the positive excess pore pressures generate in front of the T-bar at the invert level due to compression loading (see Figure 16a and b). On reversal of the displacement in the T-bar extraction pass, the suction pressures develop at the T-bar invert level because of the extension loading (see Figure 16c and d). Therefore, using a fully undrained approach - which is computationally more efficient in such a large deformation problem because there is one less nodal degree of freedom - is an appropriate choice for investigation of the undrained penetration response being simulated in this instance. However, the use of pore pressure elements is inevitable if partially drained conditions dominate the response. 


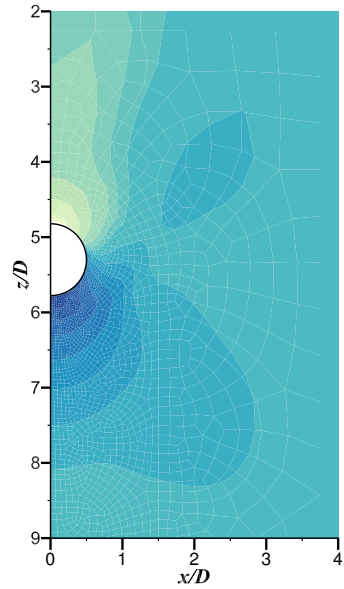

(a)

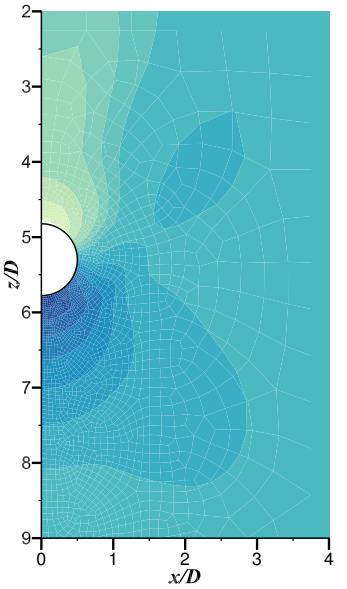

(b)

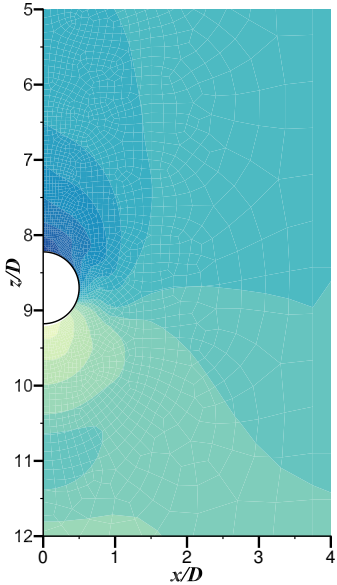

(c)

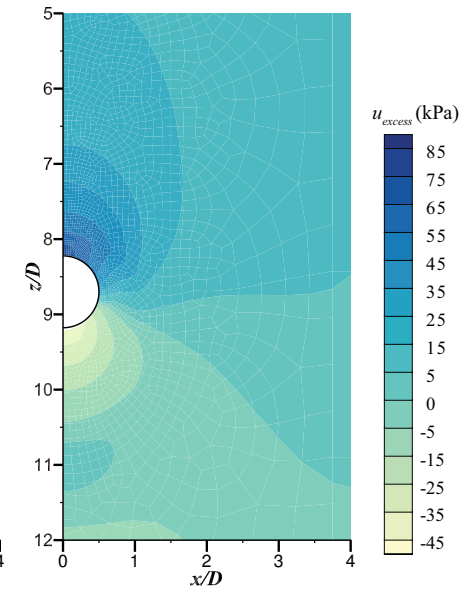

(d)

Figure 16: Distribution of the excess pore pressures $\left(u_{\text {excess }}\right)$ in T-bar cycling analyses using (a, c) normal elements $(\mathrm{CPE} 8 \mathrm{R}$ in the Abaqus Standard library) with bulk modulus and (b, d) coupled pore pressure elements (CPE8RP in the Abaqus Standard library) after T-bar advances by: (a, b) $0.3 D$ from its initial position in the first penetration pass; and (c, d) $0.3 D$ from the bottom of the penetration pass in the first extraction pass. 
Following the undrained episode, complete dissipation of the excess pore pressures was allowed, parking the T-bar at mid-depth of the cycling zone; the resulting consolidation of the soil leads to regain of the shear strength. After the re-consolidation of the soil, only a single cycle of undrained penetration (i.e total T-bar displacement of 6D) was performed. This is because the resulting highly nonlinear distribution of void ratio around the T-bar following the re-consolidation increases the propensity for interpolation errors, which results in eventual convergence problems. However, the interpolation error was straightforward to quantify and apply the drift correction in the first packet of the undrained penetration due to the void ratio being a state variable that decreases linearly with depth. The response obtained is shown in the Figure 17 as a red dashed line with triangular markers. The penetration resistance increased by about $50 \%$ compared to the remoulded resistance observed at the end of the first packet of cycles. The results derived from the second packet of cyclic T-bar penetration test [55] are also plotted in the figure as blue square markers. The actual test contained three packets of 20 cycles of undrained T-bar penetration. However, $\approx 95 \%$ strain softening occurred in the first five cycles, and therefore only the first five cycles are shown in this comparison.

Contrary to the observations made from the T-bar penetration tests at UWA [54,55], the subsequent cycle generates a practically constant resistance with no reduction in resistance during the subsequent cycle. This is because the sensitivity state variable has already reached a minimum and re-consolidation did nothing to change that. Most existing constitutive models in their current form, including that applied in this study, do not allow recovery of the sedimentation or post-sedimentation inter-particle structure of the soil that occurs due to primary and secondary consolidation, creep and thixotropy processes following the initial undrained remoulding process. As these effects cannot be observed in a standard element test, such as a triaxial test, the existing strain-softening-hardening constitutive models were not developed to take into account the aspects of behaviour that these large deformation simulations have highlighted. If this genera of problem is to be simulated accurately, new constitutive models are required that facilitate the recovery of the sensitivity state variable during dissipation and allow subsequent packets of cycles of softening to be captured. 


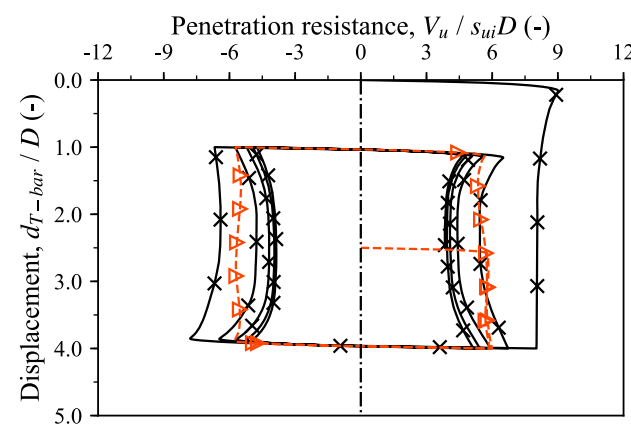

(a)

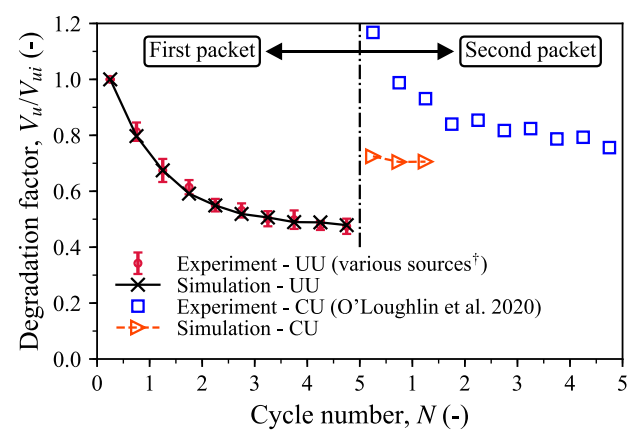

(b)

${ }^{\dagger}$ Ragni et el. 2015; O’Loughlin et al. 2020

Figure 17: Large deformation non-local simulations of cyclic undrained and consolidatedundrained T-bar penetration with $k=0.06$ and $S_{t}=2.78$.

\section{Conclusions}

This paper presented a methodology to simulate large deformation behaviour of sensitive clays in finite element analyses based on a non-local strain-softening approach. The Structured Modified Cam Clay (SMCC) constitutive model and its non-local variants have been implemented into Abaqus via a user material subroutine, or UMAT. Non-local spatial averaging was applied to the plastic strains that control the strain-softening response of the model. The mesh dependency issues of the local strain-softening model have been highlighted and the efficacy of the non-local approaches in mitigating mesh-dependency are demonstrated through a systematic series of small strain biaxial compression and large strain buried pipe uplift analyses under undrained and drained conditions. Finally, an ultra large deformation problem in the form of cyclic undrained T-bar penetration was simulated using the techniques developed. A number of new observations have been 
made through these analyses:

1. The non-local regularisation technique is not strictly necessary for the simulation of a drained biaxial compression using coupled effective stress analyses techniques. This is because the degree of strain-hardening in the response is - for the constitutive model parameters adopted greater than that of the strain-softening that is simultaneously occurring during the biaxial simulation.

2. The validity of the softening-scaling rule proposed by Galavi and Schweiger [25] for the multi-laminate constitutive model, has been confirmed as valid for the critical state based elastoplastic model adopted in this study. This finding is critical because otherwise the simulation of large scale problems involving soft clay would be practically impossible with currently available computational power.

3. Non-local regularisation techniques have been shown to mitigate mesh dependency in large deformation updated Lagrangian RITSS analyses by simulating classical problems such as pipe uplift and cyclic T-bar penetration. These analyses indicated that the strain-softening rate parameter is likely to be significantly over-estimated if element tests are used as the sole means of model calibration.

4. Existing analytical methods for determining strain-softening parameters directly from cyclic T-bar experiments are unable to predict the correct parameters for strain-softening models implemented with nonlocal regularisation. This is because these methods such as UBSPM method overestimate the accumulated shear strains, therefore a lower value of the the strain-softening rate parameter $k$ is predicted. In addition, the non-local regularisation effectively attenuates the rate of strain-softening, therefore a larger value of $k$ is required to achieve a match between the experimental data and the results of non-locally regularised FE simulations. This necessitates the development of new techniques for strain-softening parameter calibration if non-locally regularised models are to be of practical use.

5. The model developed was unable to capture the second packet of strength degradation as well as the magnitude observed in T-bar experiments by Hodder et al. [54] and O'Loughlin et al. [55]. This is because the sensitivity state variable does not recover during the dissipation process, which results in a monotonic response after the soil is fully remoulded for the first time. Accurately simulating cyclic undrained problems such 
as T-bar penetration with multiple packets of alternating remoulding cycles and dissipation requires the development of new constitutive model features that allow some recovery of sensitivity.

\section{Acknowledgements}

The first author is supported by UWA Scholarship for International Research Fees, University Postgraduate Award and Ad Hoc Top Up Scholarship. Prior to moving to the University of Cambridge, the second author was supported at the University of Western Australia by an ARC DECRA Fellowship (DE170100119).

\section{References}

[1] L. J. Sluys, Wave propagation, localisation and dispersion in softening solids, Ph.D. thesis, Delft University of Technology, Delft, The Netherlands., 1992.

[2] R. de Borst, L. J. Sluys, H. B. Mühlhaus, J. Pamin, Fundamental issues in finite element analyses of localization of deformation, Engineering Computations 10 (1993) 99-121.

[3] H. B. Mühlhaus, I. Vardoulakis, The thickness of shear hands in granular materials, Géotechnique 37 (1987) 271-283.

[4] R. de Borst, Simulation of strain localization: a reappraisal of the cosserat continuum, Engineering Computations 8 (1991) 317-332.

[5] Z. P. Bažant, Imbricate continuum and its variational derivation, Journal of Engineering Mechanics 110 (1984) 1693-1712.

[6] H. B. Mühlhaus, E. C. Alfantis, A variational principle for gradient plasticity, International Journal of Solids and Structures 28 (1991) 845 -857 .

[7] A. C. Eringen, On nonlocal plasticity, International Journal of Engineering Science 19 (1981) 14611474.

[8] G. Pijaudier-Cabot, Z. P. Bažant, Nonlocal damage theory, Journal of Engineering Mechanics 113 (1987) 1512-1533. 
[9] J. Bobiński, J. Tejchman, Modelling of concrete behaviour with a nonlocal continuum damage approach 52 (2005) 243-263.

[10] I. Einav, M. F. Randolph, Combining upper bound and strain path methods for evaluating penetration resistance, International Journal for Numerical Methods in Engineering 63 (2005) 1991-2016.

[11] M. F. Randolph, K. H. Andersen, Numerical analysis of T-bar penetration in soft clay, International Journal of Geomechanics 6 (2006) 411-420.

[12] H. Zhou, M. F. Randolph, Computational techniques and shear band development for cylindrical and spherical penetrometers in strain-softening clay, International Journal of Geomechanics 7 (2007) 287-295.

[13] H. Zhou, M. F. Randolph, Resistance of full-flow penetrometers in ratedependent and strain-softening clay, Géotechnique 59 (2009) 79-86.

[14] H. Zhou, M. F. Randolph, Numerical investigations into cycling of fullflow penetrometers in soft clay, Géotechnique 59 (2009) 801-812.

[15] U. Dayal, J. H. Allen, The effect of penetration rate on the strength of remolded clay and sand samples, Canadian Geotechnical Journal 12 (1975) 336-348.

[16] G. Biscontin, J. Pestana, Influence of peripheral velocity on vane shear strength of an artificial clay, Geotechnical Testing Journal 24 (2001) 423-429.

[17] M. F. Randolph, H. Low, H. Zhou, In situ testing for design of pipeline and anchoring systems, in: Proc. 6th Int. Conf. on Offshore Site Investigation and Geotechnics (2007), London, pp. 251-262.

[18] D. A. Bruton, D. J. White, M. Carr, J. C. Cheuk, Pipe-soil interaction with flowlines during lateral buckling and pipeline walking - the safebuck jip, in: Proc. Offshore Technol. Conf. (2008), Houston, TX, Paper OTC19589.

[19] Z. P. Bažant, M. Jirásek, Nonlocal Integral Formulations of Plasticity and Damage: Survey of Progress, Journal of Engineering Mechanics 128 (2002) 1119-1149. 
[20] D. Mašín, Comparison of predictive capabilities of selected elasto-plastic and hypoplastic models for structured clays, Soils and Foundations 49 (2009) 381-390.

[21] Y. Hu, M. F. Randolph, A practical numerical approach for large deformation problems in soil, International Journal for Numerical and Analytical Methods in Geomechanics 22 (1998) 327-350.

[22] K. H. Roscoe, J. B. Burland, On the generalized stress-strain behaviour of wet clay, Engineering Plasticity, Cambridge University Press (1968) 535-609.

[23] R. Butterfield, A natural compression law for soils (an advance on e-log p'), Géotechnique 29 (1979) 469-480.

[24] M. Taiebat, Y. F. Dafalias, R. Peek, A destructuration theory and its application to saniclay model, International Journal for Numerical and Analytical Methods in Geomechanics 34 (2010) 1009-1040.

[25] V. Galavi, H. F. Schweiger, Nonlocal multilaminate model for strain softening analysis, International Journal of Geomechanics 10 (2010) $30-44$.

[26] F. C. Summersgill, S. Kontoe, D. Potts, A comparison of the mesh dependence of the nonlocal and local strain softening methods in biaxial compression, in: Proceedings of the 8th European Conference on Numerical Methods in Geotechnical Engineering, Delft, Netherlands, pp. 289-294.

[27] M. A. Mánica, A. Gens, J. Vaunat, D. F. Ruiz, Nonlocal plasticity modelling of strain localisation in stiff clays, Computers and Geotechnics 103 (2018) $138-150$.

[28] L. Monforte, M. O. Ciantia, J. M. Carbonell, M. Arroyo, A. Gens, A stable mesh-independent approach for numerical modelling of structured soils at large strains, Computers and Geotechnics 116 (2019) 103215.

[29] R. B. J. Brinkgreve, Geomaterial models and numerical analysis of softening, Ph.D. thesis, Delft University of Technology, Netherlands., 1994. 
[30] Dassault systèmes, abaqus analysis users manual, Simulia Corp. Providence, RI, USA (2014).

[31] G. Gudehus, A. Amorosi, A. Gens, I. Herle, D. Kolymbas, D. Man, D. Muir Wood, A. Niemunis, R. Nova, M. Pastor, C. Tamagnini, G. Viggiani, The soilmodels.info project, International Journal for Numerical and Analytical Methods in Geomechanics 32 (2007) 1571 - 1572.

[32] S. Sloan, A. Abbo, D. Sheng, Refined explicit integration of elastoplastic models with automatic error control, Engineering Computations 19 (2002) 594-594.

[33] C. L. Chow, J. Mao, J. Shen, Nonlocal damage gradient model for fracture characterization of aluminum alloy, International Journal of Damage Mechanics 20 (2011) 1073-1093.

[34] J. Bobiński, FE analysis of strain localization in concrete in elastoplasticity and damage mechanics with non-local softening 10 (2006) 353375 .

[35] R. Ragni, B. Bienen, D. Wang, M. Cassidy, Modelling the effects of pauses during spudcan penetration on the further installation behaviour, 15th International Conference: The Jack-Up Platform Design, Construction and Operation. London, United Kingdom.

[36] D. P. Stewart, Lateral loading of piled bridge abutments due to embankment construction, Ph.D. thesis, The University of Western Australia, Perth, Australia., 1992.

[37] S. Chatterjee, Numerical modelling of pipe-soil interactions, Ph.D. thesis, The University of Western Australia, Perth, Australia., 2012.

[38] Y. Yan, Novel methods for characterising pipe-soil interaction forces in situ in deep water, Ph.D. thesis, The University of Western Australia, Perth, Australia., 2013.

[39] C. Vulpe, S. M. Gourvenec, A. F. Cornelius, Effect of embedment on consolidated undrained capacity of skirted circular foundations in soft clay under planar loading, Canadian Geotechnical Journal 54 (2017) $158-172$. 
[40] V. Singh, S. Chatterjee, Elastoplastic consolidation above and beneath strip anchors under uplift forces, Marine Georesources \& Geotechnology 36 (2018) 505-514.

[41] M. A. Schneider, S. A. Stanier, S. Chatterjee, D. J. White, M. F. Randolph, The parkable piezoprobe for determining $\mathrm{cv}$ and strength modelling and interpretation methods, Géotechnique 69 (2019) 458-469.

[42] F. Summersgill, S. Kontoe, D. Potts, On the use of nonlocal regularisation in slope stability problems, Computers and Geotechnics 82 (2017) $187-200$.

[43] F. C. Summersgill, Numerical modelling of stiff clay cut slopes with nonlocal strain regularisation, Ph.D. thesis, Imperial College London, London, UK., 2014.

[44] A. S. Gylland, H. P. Jostad, S. Nordal, Experimental study of strain localization in sensitive clays, Acta Geotechnica 9 (2014) 227-240.

[45] D. Muir Wood, Discussion: Heterogeneity and soil element testing, Géotechnique Letters 2 (2012) 217-219.

[46] S. Pietruszczak, Z. Mróz, Finite element analysis of deformation of strain-softening materials, International Journal for Numerical Methods in Engineering 17 (1981) 327-334.

[47] T. Marcher, Nichtlokale Modellierung der Entfestigung dichter Sande und steifer Tone, Ph.D. thesis, University of Stuttgart, Germany., 2003.

[48] D. Wang, D. J. White, M. F. Randolph, Large-deformation finite element analysis of pipe penetration and large-amplitude lateral displacement, Canadian Geotechnical Journal 47 (2010) 842856.

[49] Y. Tian, M. J. Cassidy, M. F. Randolph, D. Wang, C. Gaudin, A simple implementation of RITSS and its application in large deformation analysis, Computers and Geotechnics 56 (2014) 160 - 167.

[50] M. F. Randolph, G. T. Houlsby, The limiting pressure on a circular pile loaded laterally in cohesive soil, Géotechnique 34 (1984) 613-623. 
[51] S. Maitra, S. Chatterjee, D. Choudhury, Generalized framework to predict undrained uplift capacity of buried offshore pipelines, Canadian Geotechnical Journal 53 (2016) 1841-1852.

[52] B. M. Lehane, C. D. O'Loughlin, C. Gaudin, M. F. Randolph, Rate effects on penetrometer resistance in kaolin, Géotechnique 59 (2009) $41-52$.

[53] C. Colreavy, C. D. O'Loughlin, M. F. Randolph, Experience with a dual pore pressure element piezoball, International Journal of Physical Modelling in Geotechnics 16 (2016) 101-118.

[54] M. Hodder, D. White, M. Cassidy, An effective stress framework for the variation in penetration resistance due to episodes of remoulding and reconsolidation, Géotechnique 63 (2013) 30-43.

[55] C. D. O'Loughlin, Z. Zhou, S. A. Stanier, D. J. White, Load-controlled cyclic T-bar tests: a new method to assess effects of cyclic loading and consolidation, Géotechnique Letters 10 (2020) 7-15.

[56] H. Zhou, M. F. Randolph, Computational techniques and shear band development for cylindrical and spherical penetrometers in strain-softening clay, International Journal of Geomechanics 7 (2007) 287-295. 